TRANSACTIONS OF THE

AMERICAN MATHEMATICAL SOCIETY

Volume 362, Number 2, February 2010, Pages 859-895

S 0002-9947(09)04979-4

Article electronically published on September 18, 2009

\title{
ON CLUSTER ALGEBRAS WITH COEFFICIENTS AND 2-CALABI-YAU CATEGORIES
}

\author{
CHANGJIAN FU AND BERNHARD KELLER
}

\begin{abstract}
Building on work by Geiss-Leclerc-Schröer and by Buan-IyamaReiten-Scott we investigate the link between certain cluster algebras with coefficients and suitable 2-Calabi-Yau categories. These include the cluster categories associated with acyclic quivers and certain Frobenius subcategories of module categories over preprojective algebras. Our motivation comes from the conjectures formulated by Fomin and Zelevinsky in 'Cluster algebras IV: Coefficients'. We provide new evidence for Conjectures 5.4, 6.10, 7.2, 7.10 and 7.12 and show by an example that the statement of Conjecture 7.17 does not always hold.
\end{abstract}

\section{INTRODUCTION}

In this article, we pursue the representation-theoretic approach to FominZelevinsky's cluster algebras [19], [20, [8], 21] developed by Marsh-ReinekeZelevinsky 35, Buan-Marsh-Reineke-Reiten-Todorov 6, 7, Caldero-Chapoton and Caldero-Keller [9], 10], 11], Geiss-Leclerc-Schröer [25, [26], 23], 22] and many others; cf. the surveys [4], 31, 39], 40].

Our emphasis here is on cluster algebras with coefficients. More precisely, we investigate certain symmetric cluster algebras of geometric type with coefficients. Coefficients are of great importance both in geometric examples of cluster algebras [27], [28], 8], 41], 23] and in the study of duality phenomena [18] as shown in [21]. Following [2], we consider two types of categories which allow us to incorporate coefficients into the representation-theoretic model:

1) 2-Calabi-Yau Frobenius categories;

2) 2-Calabi-Yau 'subtriangulated' categories, i.e. full subcategories of the form ${ }^{\perp}(\Sigma \mathcal{D})$ of a 2 -Calabi-Yau triangulated category $\mathcal{C}$, where $\mathcal{D}$ is a rigid functorially finite subcategory of $\mathcal{C}$ and $\Sigma$ is the suspension functor of $\mathcal{C}$.

In both cases, we establish the link between the category and its associated cluster algebra using (variants of) cluster characters in the sense of Palu 36]. For subtriangulated categories, we use the restriction of the cluster characters constructed in 36. For Frobenius categories, we construct a suitable variant in section 3 (Theorem 3.3 .

The work of Geiss-Leclerc-Schröer [26], 23] and Buan-Iyama-Reiten-Scott [2] provides us with large classes of 2-Calabi-Yau Frobenius categories and of 2-CalabiYau subtriangulated categories which admit cluster structures in the sense of [2].

Received by the editors January 15, 2008.

2000 Mathematics Subject Classification. Primary 18E30, 16D90, 18G10.

Key words and phrases. Cluster algebra, tilting, 2-Calabi-Yau category.

(C)2009 American Mathematical Society Reverts to public domain 28 years from publication 
Our general results imply that for these classes, the 2-Calabi-Yau categories do yield 'categorifications' of the corresponding cluster algebras with coefficients (Theorems 5.4 and 6.3). As an application, we show that Conjectures 7.2, 7.10 and 7.12 of 21] hold for these cluster algebras (Theorem 5.5] and Theorem[6.3). Let us recall the statements of these conjectures:

7.2 cluster monomials are linearly independent;

7.10 different cluster monomials have different $\mathbf{g}$-vectors and the $\mathbf{g}$-vectors of the cluster variables in any given cluster form a basis of the ambient lattice;

7.12 the $\mathrm{g}$-vectors of a cluster variable with respect to two neighbouring clusters are related by a certain piecewise linear transformation (so that the gvectors equal the $\mathrm{g}^{\dagger}$-vectors of [13]).

In the case of cluster algebras with principal coefficients admitting a categorification by a 2-Calabi-Yau subtriangulated category, we obtain a representationtheoretic interpretation of the $F$-polynomial defined in section 3 of [21]; $c f$. Theorem 6.5. This interpretation implies in particular that Conjecture 5.4 of [21] holds in this case: The constant coefficient of the $F$-polynomial equals 1 . We also deduce that the multidegree of the $F$-polynomial associated with a rigid indecomposable equals the dimension vector of the corresponding module (Proposition 6.6). By combining this with recent work by Buan-Marsh-Reiten [5, cf. also [17, we obtain a counterexample to Conjecture 7.17 (and 6.11) of [21. We point out that the corresponding computations were already present in G. Cerulli's work [12. Following a suggestion by A. Zelevinsky, we show that, by assuming the existence of suitable categorifications, instead of the equality claimed in Conjecture 7.17, one does have an inequality: The multidegree of the $F$-polynomial is greater than or equal to the denominator vector (Proposition 6.8). We also show in certain cases that the transformation rule for $\mathbf{g}$-vectors predicted by Conjecture 6.10 of 21 does hold (Proposition 6.9).

Let us emphasize that our proofs for certain cluster algebras of some of the conjectures of 21] depend on the existence of suitable Hom-finite 2-Calabi-Yau categories with a cluster-tilting object. This hypothesis imposes a finiteness condition on the corresponding cluster algebra (to the best of our knowledge, it is not known how to express this condition in combinatorial terms). The construction of such 2-Calabi-Yau categories is a nontrivial problem for which we rely on [6] in the acyclic case and on 26], 23], 2] and [1] in the nonacyclic case. As A. Zelevinsky has kindly informed us, many of the conjectures of [21] will be proved in [16] in full generality building on [35] and [15].

\section{RECOLLECTIONS}

2.1. Cluster algebras. In this section, we recall the construction of cluster algebras of geometric type with coefficients from [21]. For an integer $x$, we use the notation

$$
[x]_{+}=\max (x, 0)
$$

and

$$
\operatorname{sgn}(x)= \begin{cases}-1 & \text { if } x<0 \\ 0 & \text { if } x=0 \\ -1 & \text { if } x<0\end{cases}
$$

The tropical semifield on a finite family of variables $u_{j}, j \in J$, is the abelian group (written multiplicatively) freely generated by the $u_{j}, j \in J$, endowed with 
the addition $\oplus$ defined by

$$
\prod_{j} u_{j}^{a_{j}} \oplus \prod_{j} u_{j}^{b_{j}}=\prod_{j} u_{j}^{\min \left(a_{j}, b_{j}\right)} .
$$

Let $1 \leq r \leq n$ be integers. Let $\mathbb{P}$ be the tropical semifield on the indeterminates $x_{r+1}, \ldots, x_{n}$. Let $\mathbb{Q P}$ be the group algebra on the abelian group $\mathbb{P}$. It identifies with the algebra of Laurent polynomials with rational coefficients in the variables $x_{r+1}, \ldots, x_{n}$. Let $\mathcal{F}$ be the field of fractions of the ring of polynomials with coefficients in $\mathbb{Q P}$ in $r$ indeterminates. A seed in $\mathcal{F}$ is a pair $(\tilde{B}, \mathbf{x})$ formed by

- an $n \times r$ matrix $\tilde{B}$ with integer entries whose principal part $B$ (the submatrix formed by the first $r$ rows) is antisymmetric;

- a free generating set $\mathbf{x}=\left\{x_{1}, \ldots, x_{r}\right\}$ of the field $\mathcal{F}$.

The matrix $\tilde{B}$ is called the exchange matrix and the set $\mathbf{x}$ the cluster of the seed $(\tilde{B}, \mathbf{x})$. Let $1 \leq s \leq r$ be an integer. The seed mutation in the direction $s$ transforms the seed $(\tilde{B}, \mathbf{x})$ into the seed $\mu_{s}(\tilde{B}, \mathbf{x})=\left(\tilde{B}^{\prime}, \mathbf{x}^{\prime}\right)$, where

- the entries $b_{i j}^{\prime}$ of $\tilde{B}^{\prime}$ are given by

$$
b_{i j}^{\prime}= \begin{cases}-b_{i j} & \text { if } i=s \text { or } j=s ; \\ b_{i j}+\operatorname{sgn}\left(b_{i s}\right)\left[b_{i s} b_{s j}\right]_{+} & \text {otherwise. }\end{cases}
$$

- The cluster $\mathbf{x}^{\prime}=\left\{x_{1}^{\prime}, \ldots, x_{r}^{\prime}\right\}$ is given by $x_{j}^{\prime}=x_{j}$ for $j \neq s$ whereas $x_{s}^{\prime} \in \mathcal{F}$ is determined by the exchange relation

$$
x_{s}^{\prime} x_{s}=\prod_{i=1}^{n} x_{i}^{\left[b_{i s}\right]_{+}}+\prod_{i=1}^{n} x_{i}^{\left[-b_{i s}\right]_{+}} .
$$

Mutation in a fixed direction is an involution.

Let $\mathbb{T}_{r}$ be the $r$-regular tree, whose edges are labeled by the numbers $1, \ldots, r$ so that the $r$ edges emanating from each vertex carry different labels. A cluster pattern is the assignment of a seed $\left(\tilde{B}_{t}, \mathbf{x}_{t}\right)$ to each vertex $t$ of $\mathbb{T}_{r}$ such that the seeds assigned to vertices $t$ and $t^{\prime}$ linked by an edge labeled $s$ are obtained from each other by the seed mutation $\mu_{s}$.

Fix a vertex $t_{0}$ of the $r$-regular tree $\mathbb{T}_{r}$. Clearly, a cluster pattern is uniquely determined by the initial seed $\left(\tilde{B}_{t_{0}}, x_{t_{0}}\right)$, which can be chosen arbitrarily.

Fix a seed $(\tilde{B}, \mathbf{x})$ and let $\left(\tilde{B}_{t}, \mathbf{x}_{t}\right), t \in \mathbb{T}_{r}$ be the unique cluster pattern with initial seed $(\tilde{B}, \mathbf{x})$. The clusters associated with $(\tilde{B}, \mathbf{x})$ are the sets $\mathbf{x}_{t}, t \in \mathbb{T}_{r}$. The cluster variables are the elements of the clusters. The cluster algebra $\mathcal{A}(\tilde{B})=\mathcal{A}(\tilde{B}, \mathbf{x})$ is the $\mathbb{Z P}$-subalgebra of $\mathcal{F}$ generated by the cluster variables. Its ring of coefficients is $\mathbb{Z} \mathbb{P}$. It is a 'cluster algebra without coefficients' if $r=n$ and thus $\mathbb{Z P}=\mathbb{Z}$.

2.2. Cluster algebras from ice quivers. As we have seen in the previous subsection, our cluster algebras are given by certain integer matrices $\tilde{B}$. Such matrices can also be encoded by 'ice quivers': A quiver is a quadruple $Q=\left(Q_{0}, Q_{1}, s, t\right)$, where $Q_{0}$ is a set (the set of vertices), $Q_{1}$ is a set (the set of arrows) and $s$ and $t$ are two maps $Q_{1} \rightarrow Q_{0}$ (taking an arrow to its source, respectively to its target). An ice quiver is a pair $(Q, F)$ consisting of a quiver $Q$ and a subset $F$ of its set of vertices ( $F$ is the set of frozen vertices).

Let $(Q, F)$ be an ice quiver such that the set $Q_{0}$ is the set of natural numbers from 1 to $n$, the set $Q_{1}$ is finite and the set $F$ is the set of natural numbers from $r+1$ to $n$ for some $1 \leq r \leq n$. The associated integer matrix $\tilde{B}(Q, F)$ is the $n \times r$ 
matrix whose entry $b_{i j}$ equals the number of arrows from $i$ to $j$ minus the number of arrows from $j$ to $i$. The cluster algebra with coefficients $\mathcal{A}(Q, F)$ is defined as the cluster algebra $\mathcal{A}(\tilde{B}(Q, F))$. The matrix $\tilde{B}(Q, F)$ determines the ice quiver $(Q, F)$ if

1) $Q$ does not have loops (arrows from a vertex to itself) and

2) $Q$ does not have 2-cycles (pairs of distinct arrows $\alpha, \beta$ such that $s(\alpha)=t(\beta)$ and $t(\alpha)=s(\beta))$ and

3) there are no arrows between any vertices of $F$.

Given integers $1 \leq r \leq n$, each integer matrix $\tilde{B}$ with antisymmetric principal part $B$ (formed by the first $r$ rows of $\tilde{B}$ ), is obtained as the matrix associated with a unique ice quiver satisfying these properties. The mutation of ice quivers satisfying conditions 1)-3) is defined via the mutation of the corresponding integer matrices recalled in section 2.1 .

2.3. Krull-Schmidt categories. An additive category has split idempotents if each idempotent endomorphism $e$ of an object $X$ gives rise to a direct sum decomposition $Y \oplus Z \stackrel{\rightarrow}{\rightarrow} X$ such that $Y$ is a kernel for $e$. A Krull-Schmidt category is an additive category where the endomorphism rings of indecomposable objects are local and each object decomposes into a finite direct sum of indecomposable objects (which are then unique up to isomorphism and permutation). Each Krull-Schmidt category has split idempotents. We write indec $(\mathcal{C})$ for the set of isomorphism classes of indecomposable objects of a Krull-Schmidt category $\mathcal{C}$.

Let $\mathcal{C}$ be a Krull-Schmidt category. An object $X$ of $\mathcal{C}$ is basic if every indecomposable of $\mathcal{C}$ occurs with multiplicity $\leq 1$ in $X$. In this case, $X$ is fully determined by the full additive subcategory $\operatorname{add}(X)$ whose objects are the direct factors of finite direct sums of copies of $X$. The map $X \mapsto \operatorname{add}(X)$ yields a bijection between the isomorphism classes of basic objects and the full additive subcategories of $\mathcal{C}$ which are stable under taking direct factors and only contain finitely many indecomposables up to isomorphism.

Let $k$ be an algebraically closed field. A $k$-category is a category whose morphism sets are endowed with structures of $k$-vector spaces such that the composition maps are bilinear. A $k$-category is Hom-finite if all of its morphism spaces are finite-dimensional. A $k$-linear category is a $k$-category which is additive. Let $\mathcal{C}$ be a $k$-linear Hom-finite category with split idempotents. Then $\mathcal{C}$ is a Krull-Schmidt category. Let $\mathcal{T}$ be an additive subcategory of $\mathcal{C}$ stable under taking direct factors. The quiver $Q=Q(\mathcal{T})$ of $\mathcal{T}$ is defined as follows: The vertices of $Q$ are the isomorphism classes of indecomposable objects of $\mathcal{T}$, and the number of arrows from the isoclass of $T_{1}$ to that of $T_{2}$ equals the dimension of the space of irreducible morphisms

$$
\operatorname{irr}\left(T_{1}, T_{2}\right)=\operatorname{rad}\left(T_{1}, T_{2}\right) / \operatorname{rad}^{2}\left(T_{1}, T_{2}\right),
$$

where rad denotes the radical of $\mathcal{T}$, i.e. the ideal such that $\operatorname{rad}\left(T_{1}, T_{2}\right)$ is formed by all nonisomorphisms from $T_{1}$ to $T_{2}$.

2.4. 2-Calabi-Yau triangulated categories. Let $k$ be an algebraically closed field. Let $\mathcal{C}$ be a $k$-linear triangulated category with suspension functor $\Sigma$. We assume that $\mathcal{C}$ is Hom-finite and has split idempotents. Thus, it is a Krull-Schmidt 
category. For objects $X, Y$ of $\mathcal{C}$ and an integer $i$, we define

$$
\operatorname{Ext}^{i}(X, Y)=\mathcal{C}\left(X, \Sigma^{i} Y\right) \text {. }
$$

An object $X$ of $\mathcal{C}$ is rigid if $\operatorname{Ext}^{1}(X, X)=0$.

Let $d$ be an integer. Following [42, $c f$. also [32, we define the category $\mathcal{C}$ to be $d$-Calabi-Yau if there exists a family of linear forms

$$
t_{X}: \mathcal{C}\left(X, \Sigma^{d} X\right) \rightarrow k, X \in \operatorname{obj}(\mathcal{C}),
$$

such that the bilinear forms

$$
\langle,\rangle: \mathcal{C}\left(Y, \Sigma^{d} X\right) \times \mathcal{C}(X, Y) \rightarrow k,(f, g) \mapsto t_{X}(f \circ g)
$$

are nondegenerate and satisfy

$$
\left\langle\Sigma^{p} f, g\right\rangle=(-1)^{p q}\left\langle\Sigma^{q} g, f\right\rangle
$$

for all $f$ in $\mathcal{C}\left(Y, \Sigma^{q} X\right)$ and all $g \in \mathcal{C}\left(X, \Sigma^{p} Y\right)$, where $p+q=d$.

Let us assume that $\mathcal{C}$ is 2-Calabi-Yau. A cluster-tilting subcategory of $\mathcal{C}$ is a full additive subcategory $\mathcal{T} \subset \mathcal{C}$ which is stable under taking direct factors and such that

- for each object $X$ of $\mathcal{C}$, the functors $\mathcal{C}(X, ?): \mathcal{T} \rightarrow \bmod k$ and $\mathcal{C}($ ?,$X)$ : $\mathcal{T}^{o p} \rightarrow \bmod k$ are finitely generated;

- an object $X$ of $\mathcal{C}$ belongs to $\mathcal{T}$ iff we have $\operatorname{Ext}^{1}(T, X)=0$ for all objects $T$ of $\mathcal{T}$.

A cluster-tilting object is a basic object $T$ of $\mathcal{C}$ such that $\operatorname{add}(T)$ is a clustertilting subcategory. Equivalently, an object $T$ is cluster-tilting if it is rigid and if each object $X$ satisfying $\operatorname{Ext}^{1}(T, X)=0$ belongs to add(T). The following definition is taken from section 1 of [2]. Recall that $\mathcal{C}$ is a Hom-finite $k$-linear triangulated category with split idempotents which is 2-Calabi-Yau.

Definition 2.1 ([2]). The cluster-tilting subcategories of $\mathcal{C}$ determine a cluster structure on $\mathcal{C}$ if the following hold:

0) There is at least one cluster-tilting subcategory in $\mathcal{C}$.

1) For each cluster-tilting subcategory $\mathcal{T}^{\prime}$ of $\mathcal{C}$ and each indecomposable $M$ of $\mathcal{T}^{\prime}$, there is a unique (up to isomorphism) indecomposable $M^{*}$ not isomorphic to $M$ and such that the additive subcategory $\mathcal{T}^{\prime \prime}=\mu_{M}\left(\mathcal{T}^{\prime}\right)$ of $\mathcal{C}$ with set of indecomposables

$$
\operatorname{indec}\left(\mathcal{T}^{\prime \prime}\right)=\operatorname{indec}\left(\mathcal{T}^{\prime}\right) \backslash\{M\} \cup\left\{M^{*}\right\}
$$

is a cluster-tilting subcategory.

2) In the situation of 1 ), there are triangles

$$
M^{*} \stackrel{f}{\longrightarrow} E \stackrel{g}{\longrightarrow} M \longrightarrow \Sigma M^{*} \text { and } M \stackrel{s}{\longrightarrow} E^{\prime} \stackrel{t}{\longrightarrow} M^{*} \longrightarrow \Sigma M^{*},
$$

where $g$ and $t$ are minimal right $\mathcal{T}^{\prime} \cap \mathcal{T}^{\prime \prime}$-approximations and $f$ and $s$ are minimal left $\mathcal{T}^{\prime} \cap \mathcal{T}^{\prime \prime}$-approximations.

3) For any cluster-tilting subcategory $\mathcal{T}^{\prime}$, the quiver $Q\left(\mathcal{T}^{\prime}\right)$ does not have loops nor 2-cycles.

4) We have $Q\left(\mu_{M}\left(\mathcal{T}^{\prime}\right)\right)=\mu_{M}\left(Q\left(\mathcal{T}^{\prime}\right)\right)$ for each cluster-tilting subcategory $\mathcal{T}^{\prime}$ of $\mathcal{C}$ and each indecomposable $M$ of $\mathcal{T}^{\prime}$. 
The cluster-tilting subcategory $\mathcal{T}^{\prime \prime}=\mu_{M}\left(\mathcal{T}^{\prime}\right)$ of 1) is the mutation of $\mathcal{T}^{\prime}$ at the indecomposable object $M$. The mutation of a cluster-tilting object $T$ is defined via the mutation of the cluster-tilting subcategory $\operatorname{add}(T)$.

Lemma 2.2. Suppose that the cluster-tilting subcategories determine a cluster structure on $\mathcal{C}$. Then, in the situation of condition 2) of Definition 2.1, the following hold:

a) The space $\operatorname{Ext}^{1}\left(M, M^{*}\right)$ is one-dimensional (hence, by the 2-Calabi-Yau property, so is the space $\left.\operatorname{Ext}^{1}\left(M^{*}, M\right)\right)$ and the triangles of 2) are nonsplit.

3) The multiplicity of an indecomposable $U$ of $\mathcal{T}^{\prime} \cap \mathcal{T}^{\prime \prime}$ in $E$ equals the number of arrows from $U$ to $M$ in the quiver $Q\left(\mathcal{T}^{\prime}\right)$ and that from $M^{*}$ to $U$ in $Q\left(\mathcal{T}^{\prime \prime}\right)$; the multiplicity of $U$ in $E^{\prime}$ equals the number of arrows from $M$ to $U$ in $Q\left(\mathcal{T}^{\prime}\right)$ and that from $U$ to $M^{*}$ in $Q\left(\mathcal{T}^{\prime \prime}\right)$.

Proof. a) The first triangle yields an exact sequence

$$
\mathcal{C}(M, E) \rightarrow \mathcal{C}(M, M) \rightarrow \operatorname{Ext}^{1}\left(M, M^{*}\right) \rightarrow 0 .
$$

By the absence of loops required in condition 3), each radical morphism from $M$ to $M$ factors through $E$. Since $k$ is algebraically closed, the radical is of codimension 1 in the local algebra $\mathcal{C}(M, M)$. Thus, the space $\operatorname{Ext}^{1}\left(M, M^{*}\right)$ is one-dimensional. The minimality of the approximations implies that the triangles are nonsplit. b) This follows if we combine the definition of the quivers $Q\left(\mathcal{T}^{\prime}\right)$ and $Q\left(\mathcal{T}^{\prime \prime}\right)$, with the approximation properties of $f, g, s$ and $t$.

We refer to section 1, page 11 of 2 for a list of classes of examples where this assumption holds. In particular, this list contains the cluster categories associated with finite quivers without oriented cycles and the stable categories of preprojective algebras of Dynkin quivers. We refer the reader to the surveys [4, [39, 30, 31] for more information on cluster categories and to the survey [24] for more information on stable categories of Dynkin quivers.

2.5. Cluster characters. The notion of cluster character is due to Palu [37. Under suitable assumptions, cluster characters allow one to pass from 2-Calabi-Yau categories to cluster algebras. We recall the definition and the main construction from [37. Let $k$ be an algebraically closed field and $\mathcal{C}$ a $k$-linear Hom-finite triangulated category with split idempotents which is 2-Calabi-Yau. Let $R$ be a commutative ring. A cluster character on $\mathcal{C}$ with values in $R$ is a map $\zeta: \operatorname{obj}(\mathcal{C}) \rightarrow R$ such that

1) we have $\zeta(L)=\zeta\left(L^{\prime}\right)$ if $L$ and $L^{\prime}$ are isomorphic,

2) we have $\zeta(L \oplus M)=\zeta(L) \zeta(M)$ for all objects $L$ and $M$ and

3 ) if $L$ and $M$ are objects such that $\operatorname{Ext}^{1}(L, M)$ is one-dimensional (and hence $\operatorname{Ext}^{1}(M, L)$ is one-dimensional) and

$$
L \rightarrow E \rightarrow M \rightarrow \Sigma L \text { and } M \rightarrow E^{\prime} \rightarrow L \rightarrow \Sigma M
$$

are nonsplit triangles, then we have

$$
\zeta(L) \zeta(M)=\zeta(E)+\zeta\left(E^{\prime}\right) .
$$

Assume that $\mathcal{C}$ has a cluster-tilting object $T$ which is the direct sum of $r$ pairwise nonisomorphic indecomposable summands $T_{1}, \ldots T_{r}$. In a vast generalization of 
Caldero-Chapoton's work [9, Palu has shown in 37] that there is a canonical cluster-character

$$
X_{?}^{T}: \operatorname{obj}(\mathcal{C}) \rightarrow \mathbb{Z}\left[x_{1}, \ldots, x_{r}\right], M \mapsto X_{M}^{T}
$$

such that $X_{\Sigma T_{i}}^{T}=x_{i}$ for $1 \leq i \leq r$. Let us recall Palu's construction. First we need to introduce some more notation. Let $C$ be the endomorphism algebra of $T$. Let $\bmod C$ denote the category of $k$-finite-dimensional right $C$-modules. For each $1 \leq i \leq r$, the morphism space $\mathcal{C}\left(T, T_{i}\right)$ becomes an indecomposable projective right $C$-module denoted by $P_{i}$. Its simple top is denoted by $S_{i}$. For $L$ and $M$ in $\bmod C$, define

$$
\langle L, M\rangle=\operatorname{dim} \operatorname{Hom}_{C}(L, M)-\operatorname{dim} \operatorname{Ext}_{C}^{1}(L, M)
$$

and put

$$
\langle L, M\rangle_{a}=\langle L, M\rangle-\langle M, L\rangle .
$$

By Theorem 11 of 37, the map $(L, M) \mapsto\langle L, M\rangle_{a}$ induces a well-defined bilinear form on the Grothendieck group $K_{0}(\bmod C)$. By 34, for any $X \in \mathcal{C}$, we have triangles

$$
T_{1}^{X} \rightarrow T_{0}^{X} \rightarrow X \rightarrow \Sigma T_{1}^{X} \text { and } X \rightarrow \Sigma^{2} T_{X}^{0} \rightarrow \Sigma^{2} T_{X}^{1} \rightarrow \Sigma X,
$$

where $T_{1}^{X}, T_{0}^{X}, T_{X}^{0}$ and $T_{X}^{1}$ belong to add $(T)$. The index and coindex of $X$ with respect to $T$ are defined to be the classes in $K_{0}(\operatorname{add} T)$ :

$$
\operatorname{ind}_{T}(X)=\left[T_{0}^{X}\right]-\left[T_{1}^{X}\right] \text { and } \operatorname{coind}_{T}(X)=\left[T_{X}^{0}\right]-\left[T_{X}^{1}\right] .
$$

For an object $M$ of $\mathcal{C}$, one defines

$$
X_{M}^{T}=\prod_{i=1}^{r} x_{i}^{-\left[\operatorname{coind}_{T}(M): T_{i}\right]} \sum_{e} \chi\left(G r_{e}(\mathcal{C}(T, M)) \prod_{i=1}^{r} x_{i}^{\left\langle S_{i}, e\right\rangle_{a}},\right.
$$

where $e$ runs through the positive elements of the Grothendieck group $K_{0}(\bmod C)$ and $G r_{e}(\mathcal{C}(T, M))$ denotes the variety of submodules $U$ of the right $C$-module $\mathcal{C}(T, M)$ such that the class of $U$ is $e$ and $\chi$ is the Euler characteristic (of the underlying topological space if $k=\mathbb{C}$ or of $l$-adic cohomology if $k$ is arbitrary).

2.6. From 2-CY categories to cluster algebras without coefficients. In this section, we show how certain 2-Calabi-Yau triangulated categories can be linked to cluster algebras without coefficients via cluster characters. All we need to do is to combine the results recalled in sections 2.4 and 2.5. In the main part of the paper, we will concentrate on the case where our cluster algebras do have coefficients.

Let $k$ be an algebraically closed field and $\mathcal{C}$ a Hom-finite $k$-linear 2-Calabi-Yau triangulated category with split idempotents as defined in section 2.4. Let $T$ be a cluster-tilting object in $\mathcal{C}$. Assume that $T$ is the direct sum of $r$ pairwise nonisomorphic indecomposable objects $T_{1}, \ldots, T_{r}$. Let

$$
\zeta_{T}: \operatorname{obj}(\mathcal{C}) \rightarrow \mathbb{Q}\left(x_{1}, \ldots, x_{r}\right)
$$

be Palu's cluster character associated with $T$ as recalled in section 2.5. In particular, we have

$$
\zeta_{T}\left(\Sigma T_{i}\right)=x_{i} \text { for } 1 \leq i \leq r .
$$

Now assume that the cluster-tilting subcategories define a cluster structure on $\mathcal{C}(c f$. section 2.4). A cluster-tilting object $T^{\prime}$ is reachable from $T$ if $\operatorname{add}\left(T^{\prime}\right)$ is obtained from $\operatorname{add}(T)$ by a finite sequence of mutations as defined in 2.4. A rigid object $M$ is reachable from $T$ if it lies in $\operatorname{add}\left(T^{\prime}\right)$ for a cluster-tilting object $T^{\prime}$ reachable 
from $T$. Let $Q$ be the quiver of the endomorphism algebra $C$ of $T$, or equivalently, the quiver of the category $\operatorname{add}(T)$. We consider $Q$ as an ice quiver with an empty set of frozen vertices and denote by $\mathcal{A}(Q)$ the associated cluster algebra without coefficients (defined by specializing the construction of 2.2 to the case where the set of frozen vertices is empty). It is the subalgebra of $\mathbb{Q}\left(x_{1}, \ldots, x_{r}\right)$ generated by the cluster variables.

Proposition 2.3. Assume that the above assumptions hold and in particular that the cluster-tilting subcategories define a cluster-structure on $\mathcal{C}$ (cf. section 2.4).

a) The map $M \mapsto \zeta_{T}(\Sigma M)$ induces a surjection from the set of rigid objects reachable from $T$ onto the set of cluster variables of the cluster algebra $\mathcal{A}(Q)$.

b) The surjection of a) induces a surjection from the set of cluster-tilting objects reachable from $T$ onto the set of clusters of $\mathcal{A}(Q)$.

Proof. Clearly, part a) follows from part b). Let us prove part b). Let $\mathbb{T}_{r}$ be the $r$-regular tree and let $t_{0}$ be a fixed vertex of $\mathbb{T}_{r}$. Let $B$ be the antisymmetric matrix associated with the quiver $Q$ and let $\mathbf{x}$ be the initial cluster $x_{1}, \ldots, x_{r}$. Let $\left(B_{t}, \mathbf{x}_{t}\right), t \in \mathbb{T}_{r}$, be the unique cluster pattern with initial seed $\left(B_{t_{0}}, \mathbf{x}_{t_{0}}\right)=(B, \mathbf{x})$ ( $c f$. section 2.1). To each vertex $t$ of $\mathbb{T}$, we assign a cluster-tilting object $T_{t}$ with indecomposable direct summands $T_{t, 1}, \ldots, T_{t, r}$ such that

1) we have $T_{t_{0}}=T$ and

2) if $t$ is linked to $t^{\prime}$ by an edge labeled $s$, then $T_{t^{\prime}}$ is obtained from $T_{t}$ by mutating at the summand $T_{t, s}$.

It follows from point 1) of the definition of a cluster structure that $T_{t}$ is well-defined for each vertex $t$ of $\mathbb{T}$. Moreover, it follows from point 4) of the same definition that for each vertex $t$ of $\mathbb{T}$, the antisymmetric matrix $B_{t}$ corresponds to the quiver of the category $\operatorname{add}\left(T_{t}\right)$ under the bijection of section 2.2. We claim that for each vertex $t$ of $\mathbb{T}$, the cluster character takes the shift $\Sigma T_{t, i}$ of the indecomposable direct summand $T_{t, i}$ of $T_{t}$ to the cluster variable $x_{t, i}, 1 \leq i \leq r$. Indeed, this holds for $t=t_{0}$ by equation (2.2). Now assume that it holds for some vertex $t$ and that $t$ is linked to a vertex $t^{\prime}$ by an edge labeled $s$. We know that the indecomposable summands of $T_{t^{\prime}}$ are the $T_{t^{\prime}, i}=T_{t, i}$ for $i \neq s$ and a new summand $T_{t, s}^{\prime}$ which is not isomorphic to $T_{t, s}$. By part a) of Lemma 2.2, the extension space between $T_{t, s}$ and $T_{t^{\prime}, s}$ is one-dimensional and we have the nonsplit triangles

$$
T_{t^{\prime}, s} \rightarrow E \rightarrow T_{t, s} \rightarrow \Sigma T_{t^{\prime}, s} \text { and } T_{t, s} \rightarrow E^{\prime} \rightarrow T_{t^{\prime}, s} \rightarrow \Sigma T_{t, s} .
$$

Here, the middle terms are sums of copies of the $T_{t, i}, i \neq s$, and the multiplicities are determined by the quivers of the endomorphism algebras of $T$ and $T^{\prime}$, as indicated in part b) of Lemma 2.2. More precisely, if $b_{i j}^{t}$ denotes the $(i, j)$-entry of the exchange matrix, then the summand $T_{t, i}$ occurs in $E$ with multiplicity $\left[b_{i s}^{t}\right]_{+}$and in $E^{\prime}$ with multiplicity $\left[b_{s i}^{t}\right]_{+}=\left[-b_{i s}^{t}\right]_{+}$. Now if we use points 2) and 3) of the definition of a cluster character, we see that the induction hypothesis and equation (2.1) yield the exchange relation

$$
x_{t, s} \zeta_{T}\left(\Sigma T_{t^{\prime}, s}\right)=\prod_{i=1}^{n} x_{i}^{\left[b_{i k}^{t}\right]_{+}}+\prod_{i=1}^{n} x_{i}^{\left[-b_{i k}^{t}\right]_{+}} .
$$

Thus, we have $\zeta_{T}\left(\Sigma T_{t^{\prime}, s}\right)=x_{t^{\prime}, s}$ as required. 
2.7. Frobenius categories. A Frobenius category is an exact category in the sense of Quillen [38] which has enough projectives and enough injectives and where an object is projective iff it is injective. By definition, such a category is endowed with a class of admissible exact sequences

$$
0 \rightarrow L \rightarrow M \rightarrow N \rightarrow 0 .
$$

Following [22] we will call the left morphism $L \rightarrow M$ of such a sequence an inflation, the right morphism a deflation and, sometimes, the whole sequence a conflation. Let $\mathcal{E}$ be a Frobenius category. Its associated stable category $\underline{\mathcal{E}}$ is the quotient of $\mathcal{E}$ by the ideal of morphisms factoring through a projective-injective object. It was shown by Happel [29] that $\underline{\mathcal{E}}$ has a canonical structure of a triangulated category. We have

$$
\operatorname{Ext}_{\mathcal{E}}^{i}(L, M) \stackrel{\sim}{\rightarrow} \operatorname{Ext}_{\underline{\mathcal{E}}}^{i}(L, M)
$$

for all objects $L$ and $M$ of $\mathcal{E}$ and all integers $i \geq 1$. An object $M$ of $\mathcal{E}$ is rigid if $\operatorname{Ext}_{\mathcal{E}}^{1}(M, M)=0$.

Let $k$ be an algebraically closed field and $\mathcal{E}$ a Hom-finite Frobenius category with split idempotents. Suppose that $\mathcal{E}$ is a 2-Calabi-Yau Frobenius category, i.e. its associated stable category $\mathcal{C}=\underline{\mathcal{E}}$ is 2-Calabi-Yau in the sense of section 2.4 A cluster-tilting subcategory of $\mathcal{E}$ is a full additive subcategory $\mathcal{T} \subset \mathcal{E}$ which is stable under taking direct factors and such that

- for each object $X$ of $\mathcal{E}$, the functors $\mathcal{E}(X, ?): \mathcal{T} \rightarrow \bmod k$ and $\mathcal{E}(?, X)$ : $\mathcal{T}^{o p} \rightarrow \bmod k$ are finitely generated;

- an object $X$ of $\mathcal{E}$ belongs to $\mathcal{T}$ iff we have $\operatorname{Ext}_{\mathcal{E}}^{1}(T, X)=0$ for all objects $T$ of $\mathcal{T}$.

Clearly if these conditions hold, each projective-injective object of $\mathcal{E}$ belongs to $\mathcal{T}$. A cluster-tilting object is a basic object $T$ of $\mathcal{E}$ such that $\operatorname{add}(T)$ is a cluster-tilting subcategory. Equivalently, an object $T$ is cluster-tilting if it is rigid and if each object $X$ satisfying $\operatorname{Ext}_{\mathcal{E}}^{1}(T, X)=0$ belongs to add $(T)$. The following definition is taken from section 1 of [2]. Recall that $\mathcal{E}$ is a $k$-linear Hom-finite Frobenius category with split idempotents such that the associated stable category $\mathcal{C}=\underline{\mathcal{E}}$ is 2-Calabi-Yau.

Definition 2.4 ([2]). The cluster-tilting subcategories of $\mathcal{E}$ determine a cluster structure on $\mathcal{E}$ if the following hold:

$0)$ There is at least one cluster-tilting subcategory in $\mathcal{E}$.

1) For each cluster-tilting subcategory $\mathcal{T}^{\prime}$ of $\mathcal{E}$ and each nonprojective indecomposable $M$ of $\mathcal{T}^{\prime}$, there is a unique (up to isomorphism) nonprojective indecomposable $M^{*}$ not isomorphic to $M$ and such that the additive subcategory $\mathcal{T}^{\prime \prime}=\mu_{M}\left(\mathcal{T}^{\prime}\right)$ of $\mathcal{E}$ with set of indecomposables

$$
\operatorname{indec}\left(\mathcal{T}^{\prime \prime}\right)=\operatorname{indec}\left(\mathcal{T}^{\prime}\right) \backslash\{M\} \cup\left\{M^{*}\right\}
$$

is a cluster-tilting subcategory.

2) In the situation of 1), there are conflations

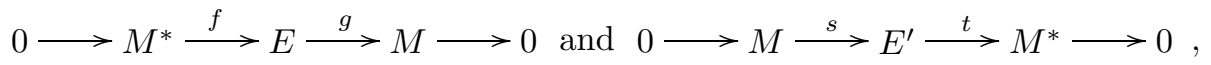

where $g$ and $t$ are minimal right $\mathcal{T}^{\prime} \cap \mathcal{T}^{\prime \prime}$-approximations, and $f$ and $s$ are minimal left $\mathcal{T}^{\prime} \cap \mathcal{T}^{\prime \prime}$-approximations.

3) For any cluster-tilting subcategory $\mathcal{T}^{\prime}$, the quiver $Q\left(\mathcal{T}^{\prime}\right)$ does not have loops nor 2-cycles. 
4) We have $Q^{\circ}\left(\mu_{M}\left(\mathcal{T}^{\prime}\right)\right)=\mu_{M}\left(Q^{\circ}\left(\mathcal{T}^{\prime}\right)\right)$ for each cluster-tilting subcategory $\mathcal{T}^{\prime}$ of $\mathcal{E}$ and each nonprojective indecomposable $M$ of $\mathcal{T}^{\prime}$, where $Q^{\circ}\left(\mathcal{T}^{\prime}\right)$ denotes the quiver obtained from $Q\left(\mathcal{T}^{\prime}\right)$ by removing all arrows between projective vertices.

The cluster-tilting subcategory $\mathcal{T}^{\prime \prime}=\mu_{M}\left(\mathcal{T}^{\prime}\right)$ of 1 ) is the mutation of $\mathcal{T}^{\prime}$ at the nonprojective indecomposable object $M$. The mutation of a cluster-tilting object $T$ is defined via the mutation of the cluster-tilting subcategory $\operatorname{add}(T)$.

Lemma 2.5. Suppose that the cluster-tilting subcategories determine a cluster structure on $\mathcal{E}$. Then, in the situation of condition 2) of Definition 2.4, the following hold:

a) The space $\operatorname{Ext}^{1}\left(M, M^{*}\right)$ is one-dimensional (hence, by the 2-Calabi-Yau property, so is the space $\operatorname{Ext}^{1}\left(M^{*}, M\right)$ ) and the conflations of 2) are nonsplit.

b) The multiplicity of an indecomposable $U$ of $\mathcal{T}^{\prime} \cap \mathcal{T}^{\prime \prime}$ in $E$ equals the number of arrows from $U$ to $M$ in the quiver $Q\left(\mathcal{T}^{\prime}\right)$ and that from $M^{*}$ to $U$ in $Q\left(\mathcal{T}^{\prime \prime}\right)$; the multiplicity of $U$ in $E^{\prime}$ equals the number of arrows from $M$ to $U$ in $Q\left(\mathcal{T}^{\prime}\right)$ and that from $U$ to $M^{*}$ in $Q\left(\mathcal{T}^{\prime \prime}\right)$.

We omit the proof of the lemma since it is entirely parallel to that of Lemma 2.2 Large classes of examples of Frobenius categories where the cluster-tilting objects define a cluster-structure are obtained in [23] and [3] ; cf. the survey 24] and Example 5.3 below. For an extension of the theory from the antisymmetric to the antisymmetrizable case, we refer to [14].

\section{Cluster characters for 2-Calabi-Yau Frobenius categories}

Let $k$ be an algebraically closed field and $\mathcal{E}$ a $k$-linear Frobenius category with split idempotents. We assume that $\mathcal{E}$ is Hom-finite and that the stable category $\mathcal{C}=\underline{\mathcal{E}}$ is 2-Calabi-Yau ( $c f$. section 2.4).

Definition 3.1. A cluster character on $\mathcal{E}$ with values in a commutative ring $R$ is a map $\zeta: \operatorname{obj}(\mathcal{E}) \rightarrow R$ such that

1) we have $\zeta(L)=\zeta\left(L^{\prime}\right)$ if $L$ and $L^{\prime}$ are isomorphic,

2) we have $\zeta(L \oplus M)=\zeta(L) \zeta(M)$ for all objects $L$ and $M$ and

3 ) if $L$ and $M$ are objects such that $\operatorname{Ext}_{\mathcal{E}}^{1}(L, M)$ is one-dimensional (and hence $\operatorname{Ext}_{\mathcal{E}}^{1}(M, L)$ is one-dimensional) and

$$
0 \rightarrow L \rightarrow E \rightarrow M \rightarrow 0 \text { and } 0 \rightarrow M \rightarrow E^{\prime} \rightarrow L \rightarrow 0
$$

are nonsplit triangles, then we have

$$
\zeta(L) \zeta(M)=\zeta(E)+\zeta\left(E^{\prime}\right) .
$$

From now on, we assume in addition that $\mathcal{E}$ contains a cluster-tilting object $T$. Using $T$ we will construct a cluster character on $\mathcal{E}$ and link it to Palu's cluster character associated with the image of $T$ in the triangulated category $\mathcal{C}=\underline{\mathcal{E}}$ (cf. section 2.5).

Let $C$ be the endomorphism algebra of $T$ (in $\mathcal{E}$ ) and $\underline{C}=\operatorname{End}_{\mathcal{C}}(T)$. Let

$$
\begin{aligned}
F & =\operatorname{Hom}_{\mathcal{E}}(T, ?): \mathcal{E} \rightarrow \bmod C, \\
G & =\operatorname{Hom}_{\mathcal{C}}(T, ?): \mathcal{C} \rightarrow \bmod \underline{C} .
\end{aligned}
$$


Let $T_{i}, 1 \leq i \leq n$, be the pairwise nonisomorphic indecomposable direct summands of $T$. We choose the numbering of the $T_{i}$ so that $T_{i}$ is projective exactly for $r<i \leq n$ for some integer $1 \leq r \leq n$. For $1 \leq i \leq n$, let $S_{i}$ be the top of the indecomposable projective $P_{i}=F T_{i}$. Note that $C$ and $\underline{C}$ are finite-dimensional $k$ algebras, so finitely presented modules are the same as finitely generated modules. As in section 4 of [34, we identify $\operatorname{Mod} \underline{C}$ with the full subcategory of $\operatorname{Mod} C$ formed by the modules without composition factors isomorphic to one of the $S_{i}$, $r<i \leq n$. Let $\mathcal{D} C$ be the unbounded derived category of the abelian category Mod $C$ of all right $C$-modules. Let per $C$ be the perfect derived category of $C$, i.e. the full subcategory of $\mathcal{D} C$ whose objects are all the complexes quasi-isomorphic to bounded complexes of finitely generated projective $C$-modules. Let $\mathcal{D}^{b}(\bmod C)$ be the bounded derived category of $\bmod C$ identified with the full subcategory of $\mathcal{D} C$ whose objects are all complexes whose total homology is finite-dimensional over $k$. As shown in section 4 of [34, we have the following embeddings:

$$
\bmod \underline{C} \hookrightarrow \operatorname{per} C \hookrightarrow \mathcal{D}^{b}(\bmod C) .
$$

We have a bilinear form

$$
\langle,\rangle: K_{0}(\operatorname{per} C) \times K_{0}\left(\mathcal{D}^{b}(\bmod C)\right) \longrightarrow \mathbb{Z}
$$

defined by

$$
\langle[P],[X]\rangle=\sum(-1)^{i} \operatorname{dim} \operatorname{Hom}_{\mathcal{D}^{b}(\bmod C)}\left(P, \Sigma^{i} X\right),
$$

where $K_{0}(\operatorname{per} C)\left(\operatorname{resp} . K_{0}\left(\mathcal{D}^{b}(\bmod C)\right)\right)$ is the Grothendieck group of $\operatorname{per} C$ (resp. $\left.\mathcal{D}^{b}(\bmod C)\right)$ and $\Sigma$ is the shift functor of $\mathcal{D}^{b}(\bmod C)$.

For arbitrary finitely generated $C$-modules $L$ and $N$, put

$$
[L, N]={ }^{0}[L, N]=\operatorname{dim}_{k} \operatorname{Hom}_{C}(L, N) \text { and }{ }^{i}[L, N]=\operatorname{dim}_{k} \operatorname{Ext}_{C}^{i}(L, N) \text { for } i \geq 1 \text {. }
$$

Let

$$
\langle L, N\rangle_{\tau}=[L, N]-{ }^{1}[L, N] \text { and }\langle L, N\rangle_{3}=\sum_{i=0}^{3}(-1)^{i}{ }^{i}[L, N]
$$

be the truncated Euler forms on the split Grothendieck group $K_{0}^{s p}(\bmod C)$. By the proposition below, if $L$ is a $\underline{C}$-module, then $\langle L, N\rangle_{3}$ only depends on the dimension vector $\underline{\operatorname{dim}} L$ in $K_{0}(\bmod C)$. We put

$$
\langle\underline{\operatorname{dim}} L, N\rangle_{3}=\langle L, N\rangle_{3} .
$$

Proposition 3.2. $\quad$ a) The restriction of the map

$$
K_{0}(\operatorname{per} C) \longrightarrow K_{0}\left(D^{b}(\bmod C)\right)=K_{0}(\bmod C)
$$

induced by the inclusion of $\operatorname{per} C$ into $\mathcal{D}^{b}(\bmod C)$ to the subgroup generated by the $\left[S_{i}\right], 1 \leq i \leq r$, is injective.

b) If $L, N$ are two $\underline{C}$-modules such that $\underline{\operatorname{dim}} L=\underline{\operatorname{dim}} N$ in $K_{0}(\bmod C)$, then

$$
\langle L, Y\rangle_{3}=\langle N, Y\rangle_{3}
$$

for each finitely generated $C$-module $Y$.

Proof. a) We need to show that for arbitrary finitely generated $\underline{C}$-modules $L, N$ with $\underline{\operatorname{dim}} L=\underline{\operatorname{dim}} N$, we have $[L]=[N]$ in $K_{0}(\operatorname{per} C)$. Let

$$
0=L_{s} \subset L_{s-1} \subset \cdots \subset L_{0}=L
$$

and

$$
0=N_{s} \subset N_{s-1} \subset \cdots \subset N_{0}=N
$$


be composition series of $L$ and $N$, respectively. By [34], we know that every $\underline{C}$ module has projective dimension at most 3 in $\bmod C$. Assume for simplicity that $L_{s-1}=S_{1}, L_{s-2} / L_{s-1}=S_{2}$. Denote by $P_{i}^{*}$ a minimal projective resolution of $S_{i}$. Then we have the following commutative diagram:

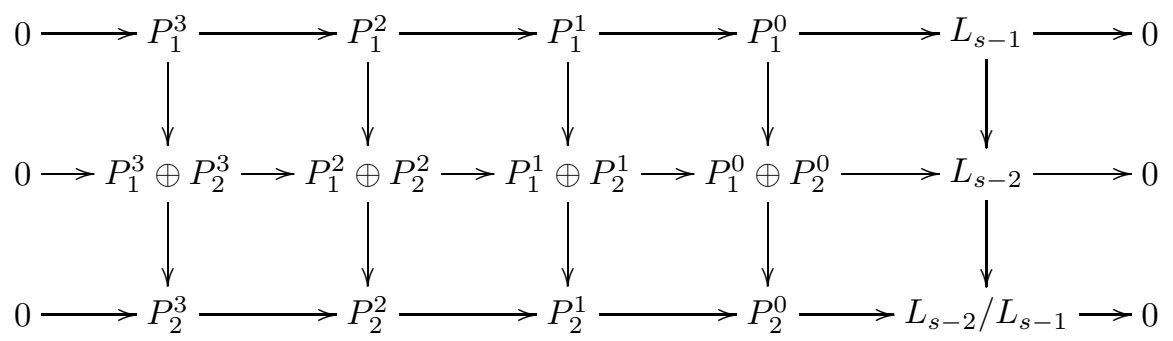

where the middle term is a projective resolution of $L_{s-2}$. In this way, we inductively construct projective resolutions for $L$ and $N$. If $m_{i}$ is the multiplicity of $S_{i}$ in the composition factors of $L$ and $N$, then we obtain projective resolutions of $L$ and $N$ of the form

$$
\begin{aligned}
& 0 \rightarrow \bigoplus_{i=1}^{r}\left(P_{i}^{3}\right)^{m_{i}} \stackrel{f_{3}}{\longrightarrow} \bigoplus_{i=1}^{r}\left(P_{i}^{2}\right)^{m_{i}} \stackrel{f_{2}}{\longrightarrow} \bigoplus_{i=1}^{r}\left(P_{i}^{1}\right)^{m_{i}} \stackrel{f_{1}}{\longrightarrow} \bigoplus_{i=1}^{r}\left(P_{i}^{0}\right)^{m_{i}} \rightarrow L \rightarrow 0, \\
& 0 \rightarrow \bigoplus_{i=1}^{r}\left(P_{i}^{3}\right)^{m_{i}} \stackrel{g_{3}}{\longrightarrow} \bigoplus_{i=1}^{r}\left(P_{i}^{2}\right)^{m_{i}} \stackrel{g_{2}}{\longrightarrow} \bigoplus_{i=1}^{r}\left(P_{i}^{1}\right)^{m_{i}} \stackrel{g_{1}}{\longrightarrow} \bigoplus_{i=1}^{r}\left(P_{i}^{0}\right)^{m_{i}} \rightarrow N \rightarrow 0 .
\end{aligned}
$$

Let $P^{L}$ (resp. $P^{N}$ ) be the projective resolution complex of $L$ (resp. $\left.N\right)$. We have $L \cong P^{L}$ and $N \cong P^{N}$ in $\operatorname{per} C$, which implies $[L]=\left[P^{L}\right]=\left[P^{N}\right]=[N]$ in $K_{0}(\operatorname{per} C)$.

b) We have

$$
\begin{aligned}
& \langle L, Y\rangle_{3}=\left\langle P^{L}, Y\right\rangle=\left\langle\left[P^{L}\right],[Y]\right\rangle, \\
& \langle N, Y\rangle_{3}=\left\langle P^{N}, Y\right\rangle=\left\langle\left[P^{N}\right],[Y]\right\rangle .
\end{aligned}
$$

By a), we have $\left[P^{L}\right]=\left[P^{N}\right]$ in $K_{0}(\operatorname{per} C)$, which implies the equality.

One should note that the truncated Euler form $\langle,\rangle_{3}$ does not descend to the Grothendieck group $K_{0}(\bmod C)$ in general (except if the global dimension of $C$ is not greater than 3); cf. Remark 3.5.

Using the bilinear forms introduced so far, for $M \in \mathcal{E}$, we define the Laurent polynomial

$$
X_{M}^{\prime}=\prod_{i=1}^{n} x_{i}^{\left\langle F M, S_{i}\right\rangle_{\tau}} \sum_{e} \chi\left(G r_{e}\left(\operatorname{Ext}_{\mathcal{E}}^{1}(T, M)\right)\right) \prod_{i=1}^{n} x_{i}^{-\left\langle e, S_{i}\right\rangle_{3}} .
$$

Here we consider $\operatorname{Ext}_{\mathcal{E}}^{1}(T, M)$ as a right $C$-module via the natural action of $C=$ $\operatorname{End}_{\mathcal{E}}(T)$ on the first argument; the sum ranges over all the elements of the Grothendieck group; for a $C$-module $L$, the notation $G r_{e}(L)$ denotes the projective variety of submodules of $L$ whose class in the Grothendieck group is $e$; for an algebraic variety $V$, the notation $\chi(V)$ denotes the Euler characteristic (of the underlying topological space of $V$ if $k=\mathbb{C}$ and of $l$-adic cohomology if $k$ is arbitrary).

Since $\mathcal{C}$ is 2-Calabi-Yau, the object $\underline{T}=\bigoplus_{i=1}^{r} T_{i}$ is a cluster-tilting object of $\mathcal{C}$. For an object $M$ of $\mathcal{C}$, put

$$
X_{M}=X_{M}^{\frac{T}{M}}
$$


where $M \mapsto X_{\bar{M}}^{\frac{T}{M}}$ is Palu's cluster character associated with the cluster-tilting object $\underline{T} ; c f$. section 2.5

The following theorem shows that $M \mapsto X_{M}^{\prime}$ is a cluster character on $\mathcal{E}$ and that, if we specialize the 'coefficients' $x_{r+1}, \ldots, x_{n}$ to 1 , it specializes to the composition of Palu's cluster character $M \mapsto X_{M}$ with the suspension functor $M \mapsto \Sigma M$. Notice that this theorem does not involve cluster algebras (but paves the way for establishing a link with cluster algebras when $\mathcal{E}$ admits a cluster structure; $c f$. Theorem 5.4 below).

Theorem 3.3. As above, let $k$ be an algebraically closed field and $\mathcal{E}$ a $k$-linear Frobenius category with split idempotents such that $\mathcal{E}$ is Hom-finite, the stable category $\mathcal{C}=\underline{\mathcal{E}}$ is 2-Calabi-Yau and $\mathcal{E}$ contains a cluster-tilting object $T$. For an object $M$ of $\mathcal{E}$, let $X_{M}^{\prime}$ and $X_{M}$ be the Laurent polynomials defined above.

a) We have $X_{T_{i}}^{\prime}=x_{i}$ for $1 \leq i \leq n$.

b) The specialization of $X_{M}^{\prime}$ at $x_{r+1}=x_{r+2}=\ldots=1$ is $X_{\Sigma M}$, where $\Sigma$ is the suspension of $\mathcal{C}$.

c) For any two objects $L$ and $M$ of $\mathcal{E}$, we have $X_{L \oplus M}^{\prime}=X_{L}^{\prime} X_{M}^{\prime}$.

d) If $L$ and $M$ are objects of $\mathcal{E}$ such that $\operatorname{Ext}_{\mathcal{E}}^{1}(L, M)$ is one-dimensional and we have nonsplit conflations

$$
0 \rightarrow L \rightarrow E \rightarrow M \rightarrow 0 \text { and } 0 \rightarrow M \rightarrow E^{\prime} \rightarrow L \rightarrow 0,
$$

then we have

$$
X_{L}^{\prime} X_{M}^{\prime}=X_{E}^{\prime}+X_{E^{\prime}}^{\prime}
$$

Proof. a) This is straightforward.

b) We have

$$
X_{\Sigma M}=\prod_{i=1}^{r} x_{i}^{-\left[\operatorname{coind}_{\underline{\underline{T}}}(\Sigma M): T_{i}\right]} \sum_{e} \chi\left(G r_{e}(G \Sigma M)\right) \prod_{i=1}^{r} x_{i}^{\left\langle S_{i}, e\right\rangle_{a}} .
$$

Now by the definition, we have

$$
G \Sigma M=\operatorname{Hom}_{\mathcal{C}}(T, \Sigma M)=\operatorname{Ext}_{\mathcal{E}}(T, M) .
$$

Therefore, we only need to show that the exponents of $x_{i}, 1 \leq i \leq r$, in the corresponding terms of $X_{\Sigma M}$ and $X_{M}^{\prime}$ are equal. There exists a triangle in $\mathcal{C}$ given by

$$
T_{M}^{1} \rightarrow T_{M}^{0} \rightarrow M \rightarrow \Sigma T_{1}
$$

with $T_{M}^{0}$ and $T_{M}^{1}$ in add $\underline{T}$. We may and will assume that this triangle is minimal, i.e. does not admit a nonzero direct factor of the form

$$
T^{\prime} \rightarrow T^{\prime} \rightarrow 0 \rightarrow \Sigma T^{\prime}
$$

Since $\mathcal{E}$ is Frobenius, we can lift this triangle to a short exact sequence in $\mathcal{E}$,

$$
0 \rightarrow T_{M}^{1} \rightarrow T_{M}^{0} \oplus P \rightarrow M \rightarrow 0,
$$

where $P$ is a projective of $\mathcal{E}$. Applying the functor $F$ to this short exact sequence, we get a projective resolution of $F M$ as a $C$-module,

$$
0 \rightarrow F T_{M}^{1} \rightarrow F\left(T_{M}^{0} \oplus P\right) \rightarrow F M \rightarrow 0 .
$$

Therefore, we have

$$
\left\langle F M, S_{i}\right\rangle_{\tau}=\left[F T_{M}^{0} \oplus F P, S_{i}\right]-\left[F T_{M}^{1}, S_{i}\right]=\left[F T_{M}^{0}, S_{i}\right]-\left[F T_{M}^{1}, S_{i}\right]
$$

for $1 \leq i \leq r$. 
On the other side, we have the following minimal triangle:

$$
\Sigma M \rightarrow \Sigma^{2} T_{M}^{1} \rightarrow \Sigma^{2} T_{M}^{0} \rightarrow \Sigma^{2} M
$$

By the definition of the coindex, we get

$$
-\left[\operatorname{coind}_{\underline{T}}(\Sigma M): T_{i}\right]=-\left[T_{M}^{1}-T_{M}^{0}: T_{i}\right]=\left\langle F M, S_{i}\right\rangle_{\tau} \text {, for } 1 \leq i \leq r .
$$

Next we will show that $\left\langle S_{i}, e\right\rangle_{a}=-\left\langle e, S_{i}\right\rangle_{3}$. Let $N$ be a $C$-module such that $\underline{\operatorname{dim}} N=e$. Note that $N$ and the $S_{i}, 1 \leq i \leq r$, are $\underline{C}$-modules and that all of them are finitely presented $C$-modules. Therefore, they lie in the perfect derived category $\operatorname{per}(C)$. Thus, we can use the relative 3-Calabi-Yau property of $\operatorname{per}(C)$ (cf. [34]) to deduce that $\left\langle S_{i}, e\right\rangle_{a}=-\left\langle e, S_{i}\right\rangle_{3}$. We have

$$
\begin{aligned}
& \operatorname{Ext}_{C}^{2}\left(N, S_{i}\right)=\operatorname{Ext}_{C}\left(S_{i}, N\right)=\operatorname{Ext}_{\underline{C}}\left(S_{i}, N\right), \\
& \operatorname{Ext}_{C}^{3}\left(N, S_{i}\right)=\operatorname{Hom}_{C}\left(S_{i}, N\right)=\operatorname{Hom}_{\underline{C}}\left(S_{i}, N\right),
\end{aligned}
$$

for $1 \leq i \leq r$. By the definition of $\left\langle S_{i}, N\right\rangle_{a}$, we have

$$
\begin{aligned}
\left\langle S_{i}, N\right\rangle_{a}= & \operatorname{dim}_{k} \operatorname{Hom}_{\underline{C}}\left(S_{i}, N\right)-\operatorname{dim}_{k} \operatorname{Ext}_{\underline{C}}\left(S_{i}, N\right)+\operatorname{dim}_{k} \operatorname{Ext}_{\underline{C}}\left(N, S_{i}\right) \\
& -\operatorname{dim}_{k} \operatorname{Hom}_{\underline{C}}\left(N, S_{i}\right) \\
= & \operatorname{dim}_{k} \operatorname{Hom}_{C}\left(S_{i}, N\right)-\operatorname{dim}_{k} \operatorname{Ext}_{C}\left(S_{i}, N\right)+\operatorname{dim}_{k} \operatorname{Ext}_{C}\left(N, S_{i}\right) \\
& -\operatorname{dim}_{k} \operatorname{Hom}_{C}\left(N, S_{i}\right) \\
= & { }^{3}\left[N, S_{i}\right]-{ }^{2}\left[N, S_{i}\right]+{ }^{1}\left[N, S_{i}\right]-\left[N, S_{i}\right] \\
= & -\left\langle N, S_{i}\right\rangle_{3} .
\end{aligned}
$$

c) This is proved in exactly the same way as Corollary 3.7 in 9 .

d) Let

$$
0 \rightarrow L \stackrel{i}{\rightarrow} E \stackrel{p}{\rightarrow} M \rightarrow 0 \text { and } 0 \rightarrow M \stackrel{i^{\prime}}{\rightarrow} E^{\prime} \stackrel{p^{\prime}}{\rightarrow} L \rightarrow 0
$$

be the nonsplit conflations in $\mathcal{E}$, and let

$$
\begin{aligned}
& \Sigma L \stackrel{G \Sigma i}{\longrightarrow} \Sigma E \stackrel{G \Sigma p}{\longrightarrow} \Sigma M \rightarrow \Sigma^{2} L, \\
& \Sigma M \stackrel{G \Sigma i^{\prime}}{\longrightarrow} \Sigma E^{\prime} \stackrel{G \Sigma p^{\prime}}{\longrightarrow} \Sigma L \rightarrow \Sigma^{2} N
\end{aligned}
$$

be the associated triangles in $\mathcal{C}$. For any classes $e, f, g$ in the Grothendieck group $K_{0}(\bmod \underline{C})$, let $X_{e, f}$ be the variety whose points are the $\underline{C}$-submodules $E \subset G \Sigma E$ such that the dimension vector of $(G \Sigma i)^{-1} E$ equals $e$ and the dimension vector of $(G \Sigma p) E$ equals $f$. Similarly, let $Y_{f, e}$ be the variety whose points are the $\underline{C}$ submodules $E \subset G \Sigma E^{\prime}$ such that the dimension vector of $\left(G \Sigma i^{\prime}\right)^{-1} E$ equals $f$ and the dimension vector of $\left(G \Sigma p^{\prime}\right) E$ equals $e$. Put

$$
\begin{gathered}
X_{e, f}^{g}=X_{e, f} \cap G r_{g}(G \Sigma E), \\
Y_{f, e}^{g}=Y_{f, e} \cap G r_{g}\left(G \Sigma E^{\prime}\right) .
\end{gathered}
$$

Since $\mathcal{C}$ is a $2-\mathrm{CY}$ triangulated category, by section 5.1 of [37] we also have

$$
\chi\left(G r_{e}(G \Sigma L) \times G r_{f}(G \Sigma M)\right)=\sum_{g} \chi\left(X_{e, f}^{g}\right)+\chi\left(Y_{f, e}^{g}\right) .
$$

Therefore, part d) is a consequence of the following lemma.

Lemma 3.4. If $X_{e, f}^{g} \neq \emptyset$, then we have the following equality:

$$
-\left\langle g, S_{i}\right\rangle_{3}+\left\langle F E, S_{i}\right\rangle_{\tau}=-\left\langle e+f, S_{i}\right\rangle_{3}+\left\langle F L, S_{i}\right\rangle_{\tau}+\left\langle F M, S_{i}\right\rangle_{\tau}, 1 \leq i \leq n .
$$


Proof. We have the following commutative diagram as in section 4 of [37:

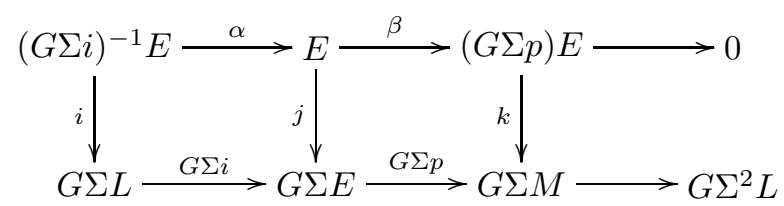

where $i, j, k$ are monomorphisms, $\beta$ is an epimorphism and $\left.[E]=g,[G \Sigma i)^{-1} E\right]=e$, $[G \Sigma p) E]=f$ in $K_{0}(\bmod C)$. One can easily show that $\operatorname{ker} G \Sigma i=\operatorname{ker} \alpha$. We have an exact sequence

$$
0 \rightarrow \operatorname{ker} \alpha \rightarrow(G \Sigma i)^{-1} E \rightarrow E \rightarrow(G \Sigma p) E \rightarrow 0 .
$$

If we apply $F=\operatorname{Hom}_{\mathcal{E}}(T, ?)$ to the short exact sequence

$$
0 \rightarrow L \rightarrow E \rightarrow M \rightarrow 0,
$$

we get the long exact sequences of $C$-modules

$$
0 \rightarrow F L \rightarrow F E \rightarrow F M \rightarrow G \Sigma L \stackrel{G \Sigma i}{\longrightarrow} G \Sigma E \rightarrow \ldots,
$$

and

$$
0 \rightarrow F L \stackrel{F i}{\longrightarrow} F E \stackrel{F p}{\longrightarrow} F M \rightarrow \text { ker } \alpha \rightarrow 0 .
$$

Since $\operatorname{ker} \alpha,(G \Sigma i)^{-1} E, E,(G \Sigma p) E$ are $\underline{C}$-modules, and the projective dimensions of $F L, F E, F M$ are not greater than 1, we can use the method of Proposition 3.2 to construct the projective resolutions and compute the truncated Euler forms. We get that

$$
\left\langle e, S_{i}\right\rangle_{3}+\left\langle f, S_{i}\right\rangle_{3}=\left\langle g, S_{i}\right\rangle_{3}+\left\langle\operatorname{ker} \alpha, S_{i}\right\rangle_{3}
$$

and

$$
\left\langle F L, S_{i}\right\rangle_{3}+\left\langle F M, S_{i}\right\rangle_{3}=\left\langle F E, S_{i}\right\rangle_{3}+\left\langle\operatorname{ker} \alpha, S_{i}\right\rangle_{3} .
$$

Note that $\left\langle F L, S_{i}\right\rangle_{3}=\left\langle F L, S_{i}\right\rangle_{\tau},\left\langle F M, S_{i}\right\rangle_{3}=\left\langle F M, S_{i}\right\rangle_{\tau}$ and $\left\langle F E, S_{i}\right\rangle_{3}=$ $\left\langle F E, S_{i}\right\rangle_{\tau}$, which implies

$$
\left\langle F L, S_{i}\right\rangle_{\tau}+\left\langle F M, S_{i}\right\rangle_{\tau}-\left\langle e+f, S_{i}\right\rangle_{3}=\left\langle F E, S_{i}\right\rangle_{\tau}-\left\langle g, S_{i}\right\rangle_{3} .
$$

Remark 3.5. If $C$ has finite global dimension, the Grothendieck group $K_{0}(\bmod C)$ has the Euler form $\langle$,$\rangle . We can then define a Laurent polynomial X_{M}^{f}$ as follows:

$$
X_{M}^{f}=\prod_{i=1}^{n} x_{i}^{\left\langle F M, S_{i}\right\rangle} \sum_{e} \chi\left(G r_{e}\left(\operatorname{Ext}_{\mathcal{E}}^{1}(T, M)\right)\right) \prod_{i=1}^{n} x_{i}^{\left\langle S_{i}, e\right\rangle} .
$$

One can show that in this case $X_{M}^{\prime}=X_{M}^{f}$. In fact, if gldim $C<\infty$, then the perfect derived category $\operatorname{per}(C)$ equals $D^{b}(\bmod C)$, and $S_{i}$ belongs to $\operatorname{per}(C)$ for all $i$. Thus, we have

$$
\left\langle S_{i}, e\right\rangle=\sum_{i=0}^{3}(-1)^{i} \operatorname{dim} \operatorname{Ext}_{C}^{i}\left(S_{i}, e\right)=-\left\langle e, S_{i}\right\rangle_{3}
$$

and $\left\langle F M, S_{i}\right\rangle_{\tau}=\left\langle F M, S_{i}\right\rangle$. The assumption that $C$ is of finite global dimension holds for the examples constructed in [2] by Proposition I.2.5 b) of [loc. cit.] and for the examples constructed in [26] by Proposition 11.5 of [loc.cit.]. 


\section{INDEX AND g-VECTOR}

4.1. Index. As in section 3 we let $k$ be an algebraically closed field and $\mathcal{E}$ a $k$ linear Frobenius category with split idempotents. We assume that $\mathcal{E}$ is Hom-finite and that the stable category $\mathcal{C}=\underline{\mathcal{E}}$ is 2-Calabi-Yau ( $c f$. section 2.4). Moreover, we assume that $\mathcal{E}$ admits a cluster-tilting object $T$ and we write $C=\operatorname{End}_{\mathcal{E}}(T)$ and $\underline{C}=\operatorname{End}_{\mathcal{C}}(T)$.

Let $\mathcal{D}(\operatorname{Mod} C)$ be the derived category of $C$-modules, $\mathcal{D}^{-}(\bmod C)$ the right bounded derived category of $\bmod C, \mathcal{H}^{-}(\mathcal{P})$ the right bounded homotopy category of finitely generated projective $C$-modules. It is well known that there is an equivalence

$$
\mathcal{H}^{-}(\mathcal{P}) \stackrel{\sim}{\longrightarrow} \mathcal{D}^{-}(\bmod C) .
$$

Proposition 4.1. For an arbitrary $\underline{C}$-module $Z$ which is also a finitely presented C-module we have a canonical isomorphism

$$
D \operatorname{Hom}_{\mathcal{D}^{-}(\bmod C)}(Z, ?) \stackrel{\sim}{\longrightarrow} \operatorname{Hom}_{\mathcal{D}^{-}(\bmod C)}(?, Z[3]) .
$$

Proof. For arbitrary $X \in \mathcal{D}^{-}(\bmod C)$, by the equivalence, we have a $P_{X} \in \mathcal{H}^{-}(\mathcal{P})$ such that $X \cong P_{X}$ in $\mathcal{D}^{-}(\bmod C)$. Assume that $P_{X}$ has the following form:

$$
\ldots \rightarrow P_{m} \rightarrow P_{m+1} \rightarrow \ldots \rightarrow P_{n-1} \rightarrow P_{n} \rightarrow 0 \rightarrow 0 \ldots
$$

Put

$$
\begin{aligned}
X_{0} & =\ldots \rightarrow 0 \rightarrow 0 \rightarrow P_{n} \rightarrow 0 \ldots, \\
X_{i} & =\ldots \rightarrow 0 \rightarrow P_{n-i} \rightarrow \ldots \rightarrow P_{n} \rightarrow 0 \ldots, \text { for } i>0 .
\end{aligned}
$$

Clearly, the complex $P_{X}$ is the direct limit of the complexes $X_{i}$. We write hocolim for the total left derived functor of the functor of taking the direct limit. Since taking direct limits over filtered systems is an exact functor, the functor hocolim is simply induced by the direct limit functor. Thus, we have $P_{X} \cong$ hocolim $X_{i}$ in $\mathcal{D}(\operatorname{Mod} C)$. Note that by Proposition 4 of [34, $Z$ belongs to per $C$; i.e. $Z$ is compact in $\mathcal{D}(\operatorname{Mod} C)$. So we have

$$
\begin{aligned}
\operatorname{Hom}_{\mathcal{D}(\operatorname{Mod} C)}(Z, X) & \cong \operatorname{Hom}_{\mathcal{D}(\operatorname{Mod} C)}\left(Z, P_{X}\right) \\
& \cong \operatorname{Hom}_{\mathcal{D}(\operatorname{Mod} C)}\left(Z, \operatorname{hocolim} X_{i}\right) \\
& \cong \operatorname{colim}_{\operatorname{Hom}}(\operatorname{Mod} C) \\
& \left(Z, X_{i}\right) .
\end{aligned}
$$

By the definition of $X_{i}$, we know that $X_{i} \in \operatorname{per} C$. Since per $C$ is a full subcategory of $\mathcal{D}(\operatorname{Mod} C)$, by the relative 3 -Calabi-Yau property of $\operatorname{per} C$, we have the following:

$$
\operatorname{colim} \operatorname{Hom}_{\mathcal{D}(\operatorname{Mod} C)}\left(Z, X_{i}\right) \cong \operatorname{colim} D \operatorname{Hom}_{\mathcal{D}(\operatorname{Mod} C)}\left(X_{i}, Z[3]\right)
$$

It is easy to see that this colimit is a stationary system; i.e. $\exists N$ such that for $i>N$, we have

$$
D \operatorname{Hom}_{\mathcal{D}(\operatorname{Mod} C)}\left(X_{i}, Z[3]\right) \cong D \operatorname{Hom}_{\mathcal{D}(\operatorname{Mod} C)}\left(X_{i+1}, Z[3]\right) .
$$

Thus, we have

$$
\begin{aligned}
\operatorname{colim} D \operatorname{Hom}_{\mathcal{D}(\operatorname{Mod} C)}\left(X_{i}, Z[3]\right) & \cong D \lim \operatorname{Hom}_{\mathcal{D}(\operatorname{Mod} C)}\left(X_{i}, Z[3]\right) \\
& \cong D \operatorname{Hom}_{\mathcal{D}(\operatorname{Mod} C)}\left(\operatorname{hocolim} X_{i}, Z[3]\right) \\
& \cong D \operatorname{Hom}_{\mathcal{D}(\operatorname{Mod} C)}\left(P_{X}, Z[3]\right) .
\end{aligned}
$$


Note that since $\mathcal{D}^{-}(\bmod C)$ is a full subcategory of $\mathcal{D}(\operatorname{Mod} C)$, we get the isomorphism

$$
D \operatorname{Hom}_{\mathcal{D}^{-}(\bmod C)}(Z, X) \stackrel{\sim}{\longrightarrow} \operatorname{Hom}_{\mathcal{D}^{-}(\bmod C)}(X, Z[3]) .
$$

For each $X \in \mathcal{E}$, there is a unique minimal conflation (up to isomorphism)

$$
0 \rightarrow T_{X}^{1} \rightarrow T_{X}^{0} \rightarrow X \rightarrow 0
$$

with $T_{X}^{0}, T_{X}^{1} \in \operatorname{add} T$. As in [37, put

$$
\operatorname{ind}_{T}(X)=\left[T_{X}^{0}\right]-\left[T_{X}^{1}\right] \text { in } K_{0}(\operatorname{add} T) .
$$

By the proof of Theorem 3.3 , we have

$$
\operatorname{ind}_{T}(X)=\sum_{i=1}^{n}\left\langle F X, S_{i}\right\rangle_{\tau}\left[T_{i}\right]
$$

The following result is easily deduced from Theorem 2.3 of [13].

Lemma 4.2. If $X$ is a rigid object of $\mathcal{E}$, then $X$ is determined up to isomorphism by $\operatorname{ind}_{T}(X)$; i.e. if $Y$ is rigid and $\operatorname{ind}_{T}(X)=\operatorname{ind}_{T}(Y)$, then $X$ is isomorphic to $Y$.

Proof. Since $\operatorname{ind}_{T}(X)=\operatorname{ind}_{T}(Y)$, we have $\operatorname{ind}_{T}(X)=\operatorname{ind}_{T}(Y)$ in the stable category $\underline{\mathcal{E}}$. By Theorem 2.3 of $\left[13\right.$, we have $X \cong Y$ in $\underline{\mathcal{E}}$. Thus, there are $\mathcal{E}$-projectives $P_{X}$ and $P_{Y}$ such that $X \oplus P_{X} \cong Y \oplus P_{Y}$ in $\mathcal{E}$. For the minimal right $T$-approximation of $X \oplus P_{X}$,

$$
0 \rightarrow T^{1} \rightarrow T^{0} \rightarrow X \oplus P_{X} \rightarrow 0,
$$

we have $\operatorname{ind}_{T}\left(X \oplus P_{X}\right)=\operatorname{ind}_{T}\left(Y \oplus P_{Y}\right)=\left[T^{0}\right]-\left[T^{1}\right]$. Note that

$$
\operatorname{ind}_{T}(X)=\operatorname{ind}_{T}\left(X \oplus P_{X}\right)-\left[P_{X}\right]=\operatorname{ind}_{T}\left(Y \oplus P_{Y}\right)-\left[P_{Y}\right]=\operatorname{ind}_{T}(Y),
$$

which implies $\left[P_{X}\right]=\left[P_{Y}\right]$ in $K_{0}(\operatorname{add} T)$. Thus, we have $P_{X} \cong P_{Y}$ and $X \cong Y$ in $\mathcal{E}$.

4.2. g-vector. Let us recall the definition of $\mathbf{g}$-vectors from section 7 of 21. Let $1<r \leq n$ be integers. Let $\tilde{B}=\left(\tilde{b}_{i j}\right)$ be an $n \times r$ matrix with integer entries, whose principal part $B$ (i.e. the submatrix formed by the first $r$ rows) is antisymmetric. Let $\mathcal{A}(\tilde{B})$ be the cluster algebra with coefficients associated with $\tilde{B} ; c f$. the end of section 2.1. Let $z$ be an element of $\mathcal{A}(\tilde{B})$. Suppose that we can write $z$ as

$$
z=R\left(\widehat{y}_{1}, \ldots, \widehat{y}_{r}\right) \prod_{i=1}^{n} x_{i}^{g_{i}}, \text { where } \widehat{y}_{j}=\prod_{i=1}^{n} x_{i}^{\tilde{b}_{i j}}
$$

where $R\left(\widehat{y}_{1}, \ldots, \widehat{y}_{r}\right)$ is a primitive rational polynomial. If $\operatorname{rank} \tilde{B}=r$, then the $\mathrm{g}$-vector of $z$ is defined by

$$
g(z)=\left(g_{1}, \ldots, g_{r}\right) .
$$

Note that $\operatorname{rank} \tilde{B}=r$ implies that the $\mathbf{g}$-vector is well-defined.

As in the previous section, we let $k$ be an algebraically closed field and $\mathcal{E}$ a $k$ linear Frobenius category with split idempotents. We assume that $\mathcal{E}$ is $\mathrm{Hom}$-finite and that the stable category $\mathcal{C}=\underline{\mathcal{E}}$ is 2-Calabi-Yau ( $c f$. section 2.4). Moreover, we assume that $\mathcal{E}$ admits a cluster-tilting object $T$ and we write $C=\operatorname{End}_{\mathcal{E}}(T)$ and $\underline{C}=\operatorname{End}_{\mathcal{C}}(T)$. Let $T_{1}, T_{2}, \ldots, T_{n}$ be the pairwise nonisomorphic indecomposable direct summands of $T$ numbered in such a way that $T_{i}$ is projective iff $r<i \leq n$. We define $B(T)=\left(b_{i j}\right)_{n \times n}$ to be the antisymmetric matrix associated with the 
quiver of the endomorphism algebra of $T$. Let $B(T)^{0}$ be the submatrix formed by the first $r$ columns of $B(T)$. We suppose that we have rank $B(T)^{0}=r$. In analogy with the definition of $\mathbf{g}$-vectors in a cluster algebra, for $M \in \mathcal{E}$, if we can write $X_{M}^{\prime}$ as

$$
X_{M}^{\prime}=R\left(\widehat{y}_{1}, \ldots, \widehat{y}_{r}\right) \prod_{i=1}^{n} x_{i}^{g_{i}}, \text { where } \widehat{y}_{j}=\prod_{i=1}^{n} x_{i}^{b_{i j}},
$$

where $R\left(\widehat{y}_{1}, \ldots, \widehat{y}_{r}\right)$ is a primitive rational polynomial, then we define the $\mathbf{g}$-vector $g_{T}\left(X_{M}^{\prime}\right)$ of $M$ with respect to $T$ to be

$$
g_{T}\left(X_{M}^{\prime}\right)=\left(g_{1}, \ldots, g_{r}\right) .
$$

As in the cluster algebra case, this is well-defined since rank $B(T)^{0}=r$.

Proposition 4.3. Assume that rank $B(T)^{0}=r$. For arbitrary $M \in \mathcal{E}$, the $\mathbf{g}$-vector $g_{T}\left(X_{M}^{\prime}\right)$ is well-defined and its $i$-th coordinate is given by

$$
g_{T}\left(X_{M}^{\prime}\right)(i)=\left[\operatorname{ind}_{T}(M): T_{i}\right], 1 \leq i \leq r .
$$

Proof. By the relative 3 -Calabi-Yau property of $\mathcal{D}^{-}(\bmod C)$, for $1 \leq i \leq n, 1 \leq$ $j \leq r$, we have

$$
\begin{aligned}
\left\langle S_{i}, S_{j}\right\rangle_{3} & =\left[S_{i}, S_{j}\right]-{ }^{1}\left[S_{i}, S_{j}\right]+{ }^{2}\left[S_{i}, S_{j}\right]-{ }^{3}\left[S_{i}, S_{j}\right] \\
& =\left[S_{i}, S_{j}\right]-{ }^{1}\left[S_{i}, S_{j}\right]+{ }^{1}\left[S_{j}, S_{i}\right]-\left[S_{j}, S_{i}\right] \\
& ={ }^{1}\left[S_{j}, S_{i}\right]-{ }^{1}\left[S_{i}, S_{j}\right] \\
& =b_{i j},
\end{aligned}
$$

where the last equality follows from the definition of $B(T)$. Recall the definition of $X_{M}^{\prime}$ :

$$
X_{M}^{\prime}=\prod_{i=1}^{n} x_{i}^{\left\langle F M, S_{i}\right\rangle_{\tau}} \sum_{e} \chi\left(G r_{e}\left(\operatorname{Ext}_{\mathcal{E}}^{1}(T, M)\right)\right) \prod_{i=1}^{n} x_{i}^{-\left\langle e, S_{i}\right\rangle_{3}} .
$$

Let $e$ be the dimension vector of a $C$-submodule of $\operatorname{Ext}_{\mathcal{E}}^{1}(T, M)$ and $e_{j}$ its $j$-th coordinate in the basis of the $S_{i}, 1 \leq i \leq n$. Then we have

$$
-\left\langle e, S_{i}\right\rangle_{3}=-\sum_{j=1}^{r} e_{j}\left\langle S_{j}, S_{i}\right\rangle_{3}=\sum_{j=1}^{r} b_{i j} e_{j} .
$$

Therefore, we get

$$
\prod_{i=1}^{n} x_{i}^{-\left\langle e, S_{i}\right\rangle_{3}}=\prod_{i=1}^{n} x_{i}^{\sum_{j=1}^{r} b_{i j} e_{j}}=\prod_{j=1}^{r} \widehat{y}_{j}{ }^{e_{j}} .
$$

Thus, we can write

$$
X_{M}^{\prime}=\prod_{i=1}^{n} x_{i}^{\left\langle F M, S_{i}\right\rangle_{\tau}}\left(\sum_{e} \chi\left(G r_{e}\left(\operatorname{Ext}_{\mathcal{E}}^{1}(T, M)\right)\right) \prod_{j=1}^{r} \widehat{y}_{j}{ }^{e_{j}}\right) .
$$

The polynomial

$$
R\left(\widehat{y}_{1}, \ldots, \widehat{y}_{r}\right)=\sum_{e} \chi\left(G r_{e}\left(\operatorname{Ext}_{\mathcal{E}}^{1}(T, M)\right)\right) \prod_{j=1}^{r} \widehat{y}_{j}{ }^{e_{j}}
$$

is primitive since it has constant term 1 . Thus, by definition, we have $g_{T}\left(X_{M}^{\prime}\right)(i)=$ $\left\langle F M, S_{i}\right\rangle_{\tau}=\left[\operatorname{ind}_{T}(M): T_{i}\right]$. 
Corollary 4.4. As above, let $\mathcal{E}$ be a Hom-finite $k$-linear Frobenius category such that its stable category $\mathcal{C}=\underline{\mathcal{E}}$ is 2-Calabi-Yau and assume that

- $\mathcal{E}$ admits a cluster-tilting object $T$ with indecomposable direct summands $T_{1}, \ldots, T_{n}$ numbered in such a way that $T_{i}$ is projective iff $r<i \leq n$, where $1<r \leq n$ is an integer;

- the first $r$ columns of the antisymmetric matrix $B(T)$ associated with the quiver of the algebra $C=\operatorname{End}_{\mathcal{E}}(T)$ are linearly independent.

Then the following hold.

a) The map $M \mapsto X_{M}^{\prime}$ induces an injection from the set of isomorphism classes of nonprojective rigid indecomposables of $\mathcal{E}$ into the set $\mathbb{Q}\left(x_{1}, \ldots, x_{n}\right)$.

b) Let $I$ be a finite set and $T^{i}, i \in I$, cluster-tilting objects of $\mathcal{E}$. Suppose that for each $i \in I$, we are given an object $M_{i}$ which belongs to add $T^{i}$ and does not have nonzero projective direct factors. If the $M_{i}$ are pairwise nonisomorphic, then the $X_{M_{i}}^{\prime}$ are linearly independent.

Proof. a) clearly follows from b). Let us prove b). First, we will show that we can assign a degree to each $x_{i}$ such that for every $1 \leq i \leq r$ the degree of $\widehat{y}_{i}$ is 1 .

Indeed, it suffices to put $\operatorname{deg}\left(x_{i}\right)=k_{i}$, where the $k_{i}$ are rationals such that we have

$$
\left(k_{1}, k_{2}, \ldots, k_{n}\right) B(T)^{0}=(1,1, \ldots, 1) .
$$

Since rank $B(T)^{0}=r$, this equation does admit a solution. Thus, the term of strictly minimal total degree in $X_{M_{j}}^{\prime}$ is

$$
\prod_{i=1}^{n} x_{i}^{\left[\operatorname{ind}_{T}\left(M_{j}\right): T_{i}\right]} .
$$

Suppose that the $X_{M_{i}}^{\prime}$ are linearly dependent; i.e. there is a nonempty subset $I^{\prime}$ of $I$ and rationals $c_{i}, i \in I^{\prime}$, which are all nonzero such that

$$
\sum_{i \in I^{\prime}} c_{i} X_{M_{i}}^{\prime}=0 .
$$

If we consider the terms of minimal total degree of the polynomial above, we find

$$
\sum_{j \in I^{\prime \prime}} c_{j} \prod_{i=1}^{n} x_{i}^{\left[\operatorname{ind}_{T}\left(M_{j}\right): T_{i}\right]}=0
$$

for some nonempty subset $I^{\prime \prime}$ of $I$. Since the $M_{j}$ are all pairwise nonisomorphic, Lemma 4.2 implies that the indices ind ${ }_{T}\left(M_{j}\right)$ are all distinct. Thus, the monomials $\prod_{i=1}^{n} x_{i}^{\left[\operatorname{ind}_{T}\left(M_{j}\right): T_{i}\right]}$ are linearly independent, a contradiction.

Remark 4.5. If the algebra $C$ has finite global dimension, then the condition rank $B(T)^{0}=r$ is superfluous. Indeed, let $A$ be the Cartan matrix of $C$. Then $B(T)^{0}$ is the submatrix formed by the first $r$ columns of the invertible matrix $A^{-t}$.

Next we will investigate the relation between the indices of an exchange pair.

Recall that $F$ is the functor $\operatorname{Hom}_{\mathcal{E}}(T, ?): \mathcal{E} \rightarrow \bmod C$. A conflation of $\mathcal{E}$,

$$
0 \rightarrow X \rightarrow Y \rightarrow Z \rightarrow 0,
$$

is F-exact if

$$
0 \rightarrow F X \rightarrow F Y \rightarrow F Z \rightarrow 0
$$


is exact in $\bmod C$. The $F$-exact sequences define a new exact structure on the additive category $\mathcal{E}$. For each $X$, we have an $F$-exact conflation

$$
0 \rightarrow T_{1} \rightarrow T_{0} \rightarrow X \rightarrow 0 .
$$

This shows that $\mathcal{E}$ endowed with the $F$-exact sequences has enough projectives and that its subcategory of projectives is add $T$. Moreover, if we denote by $\operatorname{Ext}_{F}^{i}(X, Z)$ the $i$-th extension groups of the category $\mathcal{E}$ endowed with the $F$-exact sequences, then $\operatorname{Ext}_{F}^{1}(X, Z)$ is the cohomology at $\operatorname{Hom}_{\mathcal{E}}\left(T_{1}, Z\right)$ of the complex

$$
0 \rightarrow \operatorname{Hom}_{\mathcal{E}}(X, Z) \rightarrow \operatorname{Hom}_{\mathcal{E}}\left(T_{0}, Z\right) \rightarrow \operatorname{Hom}_{\mathcal{E}}\left(T_{1}, Z\right) \rightarrow 0 \rightarrow \ldots
$$

Lemma 4.6. For $X, Z \in \mathcal{E}$, there is a functorial isomorphism

$$
\operatorname{Ext}_{F}^{i}(X, Z) \stackrel{\sim}{\longrightarrow} \operatorname{Ext}_{C}^{i}(F X, F Z) .
$$

Proof. Clearly, the derived functor

$$
\mathbb{L} F: \mathcal{D}^{b}(\mathcal{E}) \rightarrow \mathcal{D}^{b}(\bmod C)
$$

is fully faithful. Thus, $\operatorname{Ext}_{F}^{i}(X, Z) \stackrel{\sim}{\longrightarrow} \operatorname{Ext}_{C}^{i}(F X, F Z)$.

Now Proposition 15.4 of [23] still holds in our general setting.

Proposition 4.7. Let $T$ and $R$ be cluster-tilting objects of $\mathcal{E}$. Let

$$
\eta^{\prime}: 0 \rightarrow R_{k} \rightarrow R^{\prime} \rightarrow R_{k}^{*} \rightarrow 0, \eta^{\prime \prime}: 0 \rightarrow R_{k}^{*} \rightarrow R^{\prime \prime} \rightarrow R_{k} \rightarrow 0
$$

be the two exchange sequences associated to an indecomposable direct summand $R_{k}$ of $R$ which is not $\mathcal{E}$-projective. Then exactly one of $\eta^{\prime}$ and $\eta^{\prime \prime}$ is F-exact. Moreover, we have

$\underline{\operatorname{dim}} \operatorname{Hom}_{\mathcal{E}}\left(T, R_{k}\right)+\underline{\operatorname{dim}} \operatorname{Hom}_{\mathcal{E}}\left(T, R_{k}^{*}\right)=\max \left\{\underline{\operatorname{dim}} \operatorname{Hom}_{\mathcal{E}}\left(T, R^{\prime}\right), \underline{\operatorname{dim}} \operatorname{Hom}_{\mathcal{E}}\left(T, R^{\prime \prime}\right)\right\}$.

Proof. Using Lemma 4.6, the proof is the same as that of proposition 15.4 in [23].

Corollary 4.8. Under the assumptions of the above proposition, put

Then we have

$$
\begin{aligned}
& I^{\prime}=\operatorname{ind}_{T}\left(R^{\prime}\right)-\operatorname{ind}_{T}\left(R_{k}\right), \\
& I^{\prime \prime}=\operatorname{ind}_{T}\left(R^{\prime \prime}\right)-\operatorname{ind}_{T}\left(R_{k}\right) .
\end{aligned}
$$

$$
\operatorname{ind}_{T}\left(R_{k}^{*}\right)=\left\{\begin{array}{l}
I^{\prime}, \text { if } \underline{\operatorname{dim}} F I^{\prime} \geq \underline{\operatorname{dim}} F I^{\prime \prime}, \\
I^{\prime \prime}, \text { if } \underline{\operatorname{dim}} F I^{\prime} \leq \underline{\operatorname{dim}} F I^{\prime \prime},
\end{array}\right.
$$

and exactly one of these cases occurs. Let $h(i)=\left[\operatorname{ind}_{T}\left(R^{\prime}\right)-\operatorname{ind}_{T}\left(R^{\prime \prime}\right): T_{i}\right]$, for $1 \leq i \leq n$. Then $h$ is a linear combination of the columns of $B(T)^{0}$.

Proof. The first part follows from Proposition 4.7 directly, because the index is additive on $F$-exact sequences.

Since $\left(R_{k}, R_{k}^{*}\right)$ is an exchange pair, we have

$$
X_{R_{k}}^{\prime} X_{R_{k}^{*}}^{\prime}=X_{R^{\prime}}^{\prime}+X_{R^{\prime \prime}}^{\prime}
$$

For simplicity, we write

$$
H_{M}=\sum_{e} \chi\left(G r_{e}\left(\operatorname{Ext}_{\mathcal{E}}(T, M)\right)\right) \prod_{i=1}^{r} \widehat{y}_{i} e_{i}
$$


for $X_{M}^{\prime}$. By Proposition 4.3, we have

$$
\prod_{i=1}^{n} x_{i}^{\left[\operatorname{ind}_{T}\left(R_{k}\right)+\operatorname{ind}_{T}\left(R_{k}^{*}\right): T_{i}\right]} H_{R_{k}} H_{R_{k}^{*}}=\prod_{i=1}^{n} x_{i}^{\left[\operatorname{ind}_{T}\left(R^{\prime}\right): T_{i}\right]} H_{R^{\prime}}+\prod_{i=1}^{n} x_{i}^{\left[\operatorname{ind}_{T}\left(R^{\prime \prime}\right): T_{i}\right]} H_{R^{\prime \prime}} .
$$

Assume that $\operatorname{ind}_{T}\left(R_{k}^{*}\right)=\operatorname{ind}_{T}\left(R^{\prime}\right)-\operatorname{ind}_{T}\left(R_{k}\right)$. We have

$$
H_{R_{k}} H_{R_{k}^{*}}-H_{R^{\prime}}=\prod_{i=1}^{n} x_{i}^{\left[\operatorname{ind}_{T}\left(R^{\prime \prime}\right)-\operatorname{ind}_{T}\left(R^{\prime}\right): T_{i}\right]} H_{R^{\prime \prime}}
$$

By comparing the minimal total degree we get that $\prod_{i=1}^{n} x_{i}^{\left[\operatorname{ind}_{T}\left(R^{\prime \prime}\right)-\operatorname{ind}_{T}\left(R^{\prime}\right): T_{i}\right]}$ is a monomial in $\widehat{y}_{i}, 1 \leq i \leq r$, which implies the result.

\section{Frobenius 2-Calabi-Yau realizations}

Recall the bijection defined in section 2.2 between antisymmetric integer $n \times n$ matrices and finite quivers without loops or 2 -cycles with vertex set $\{1,2, \ldots, n\}$ : the quiver $Q$ corresponds to the matrix $B$ iff $b_{i j}>0$ exactly when there are arrows from $i$ to $j$ in $Q$ and in this case their number is $b_{i j}$.

We call an $n \times n$ antisymmetric integer matrix $B$ acyclic if the corresponding quiver $Q$ does not have oriented cycles. Two matrices $B$ and $B^{\prime}$ are called mutation equivalent if we can obtain $B^{\prime}$ from $B$ by a series of matrix mutations followed by conjugation with a permutation matrix.

Let $0 \leq r<n$ be positive integers and let $(Q, F)$ be an ice quiver ( $c f$. section 2.2) with vertex set $Q_{0}=\{1, \ldots, n\}$ and set of frozen vertices $F=\{r+1, \ldots, n\}$. We define $\tilde{B}$ to be the $n \times r$ matrix formed by the first $r$ columns of the skew-symmetric matrix associated with $Q$ and we let $\mathcal{A}(Q, F)=\mathcal{A}(\tilde{B})$ be the cluster algebra with coefficients associated with $\tilde{B} ; c f$. sections 2.1 and 2.2

Definition 5.1. A Frobenius 2-Calabi-Yau realization of the cluster algebra $\mathcal{A}(\tilde{B})$ is a Frobenius category $\mathcal{E}$ with a cluster-tilting object $T$ as in section 3 such that

1) $\mathcal{E}$ has a cluster structure in the sense of $[2$; cf. section 2.7

2) $T$ has exactly $n$ indecomposable pairwise nonisomorphic summands $T_{1}, T_{2}$, $\ldots, T_{n}$ and among these, precisely $T_{r+1}, \ldots, T_{n}$ are projectives.

3) The matrix $\tilde{B}$ equals the matrix formed by the first $r$ columns of the antisymmetric matrix associated with the quiver of the endomorphism algebra of $T$ in $\mathcal{E}$.

Remark 5.2. Suppose we have a Frobenius 2-CY realization of a cluster algebra $\mathcal{A}(Q, F)$ as above. Let $1 \leq s \leq r$. Then by Lemma $2.5 \mathrm{~b}$ ), we have conflations

$$
\begin{aligned}
& 0 \rightarrow T_{s}^{*} \rightarrow E \rightarrow T_{s} \rightarrow 0, \\
& 0 \rightarrow T_{s} \rightarrow E^{\prime} \rightarrow T_{s}^{*} \rightarrow 0 .
\end{aligned}
$$

Here the middle terms are the sums

$$
E=\bigoplus_{b_{i s}>0} T_{i}^{b_{i s}}, E^{\prime}=\bigoplus_{b_{i s}<0} T_{i}^{-b_{i s}} .
$$

Therefore, none of the first $r$ vertices of $Q$ can be a source or a sink. 
Example 5.3. All quivers obtained from Theorem 2.3 of [23] and, more generally, from Theorem II.4.1 of [2] admit Frobenius 2-Calabi-Yau realizations. We illustrate this for the following specific case taken from section II.4 of [loc. cit.]. Let $\Delta$ be the graph

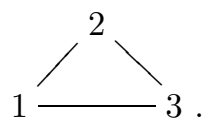

Let $\Lambda$ be the completion of the preprojective algebra of $\Delta$ and $W$ the Weyl group associated with $\Delta$. Let $w$ be the element of $W$ given by the reduced word $s_{2} s_{1} s_{2} s_{3} s_{2}$. Let $e_{i}, i=1,2,3$, be the primitive idempotents corresponding to the vertices of $\Delta$. Let $I_{i}=\Lambda\left(1-e_{i}\right) \Lambda$. By Theorem II.2.8 of 2 , the category $\operatorname{Sub} \Lambda / I_{w}$ formed by all $\Lambda$-submodules of finite direct sums of copies of $\Lambda / I_{w}$ is a Frobenius category whose associated stable category is 2-Calabi-Yau; moreover, it contains the cluster-tilting object

$$
T=\Lambda / I_{2} \oplus \Lambda / I_{2} I_{1} \oplus \Lambda / I_{2} I_{1} I_{2} \oplus \Lambda / I_{2} I_{1} I_{2} I_{3} \oplus \Lambda / I_{w} .
$$

According to Proposition II.1.11 of [loc. cit.], in this decomposition, each direct factor differs from the preceding one by one indecomposable direct summand $T_{i}, 1 \leq$ $i \leq 5$, and among these, exactly $T_{3}, T_{4}$ and $T_{5}$ are projective-injective. Moreover, by Theorem II.4.1 of [loc. cit.], the quiver of the cluster-tilting object is

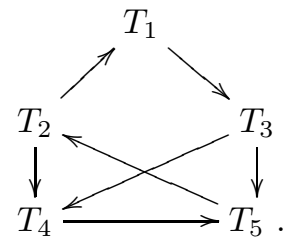

Using Theorem I.1.6 of [2], one can easily show that the category $\operatorname{Sub} \Lambda / I_{w}$ is a Frobenius 2-Calabi-Yau realization of the cluster algebra $\mathcal{A}(\tilde{B})$ given by the matrix

$$
\tilde{B}=\left(\begin{array}{cc}
0 & -1 \\
1 & 0 \\
-1 & 0 \\
0 & -1 \\
0 & 1
\end{array}\right) \text {. }
$$

We return to the general setup. Following [13] we define a cluster-tilting object $T^{\prime}$ of $\mathcal{E}$ to be reachable from $T$ if it is obtained from $T$ by a finite sequence of mutations. We define an indecomposable rigid object $M$ to be reachable from $T$ if it occurs as a direct factor of a cluster-tilting object reachable from $T$.

Theorem 5.4. Let $1<r \leq n$ be integers and $\mathcal{A}(\tilde{B})$ the cluster algebra with coefficients associated with an initial $n \times r$ matrix $\tilde{B}$ of maximal rank. Suppose that $\mathcal{A}(\tilde{B})$ admits a Frobenius $2-C Y$ realization $\mathcal{E}$ with cluster-tilting object $T$.

a) The map $M \mapsto X_{M}^{\prime}$ induces a bijection from the set of isomorphism classes of indecomposable rigid nonprojective objects of $\mathcal{E}$ reachable from $T$ onto the set of cluster variables of $\mathcal{A}(\tilde{B})$. Under this bijection, the cluster-tilting objects reachable from $T$ correspond to the clusters of $\mathcal{A}(\tilde{B})$.

b) The map $M \mapsto \operatorname{ind}_{T}(M)$ is a bijection from the set of isomorphism classes of indecomposable rigid nonprojective objects of $\mathcal{E}$ reachable from $T$ onto the set of $\mathbf{g}$-vectors of cluster variables of $\mathcal{A}(\tilde{B})$. 
Proof. a) It follows from Theorem 3.3 c) that $X_{M}^{\prime}$ is a cluster variable for each indecomposable rigid $M$ reachable from $T$ and from the existence of a cluster structure on $\mathcal{E}$ that the map $M \mapsto X_{M}^{\prime}$ is a surjection onto the set of cluster variables. The injectivity of the map $M \mapsto X_{M}^{\prime}$ follows from Lemma 4.2 and Proposition 4.3. The second statement follows from the first one and the fact that $\mathcal{E}$ has a cluster structure. b) The map is injective by Lemma 4.2. It is surjective thanks to part a) and Proposition 4.3 .

Theorem 5.5. Let $1<r \leq n$ be integers and $\mathcal{A}(\tilde{B})$ the cluster algebra with coefficients associated with an initial $n \times r$ matrix $\tilde{B}$ of maximal rank. Suppose that $\mathcal{A}(\tilde{B})$ admits a Frobenius $2-C Y$ realization $\mathcal{E}$ with cluster tilting object $T$.

a) Conjecture 7.2 of 21 holds for $\mathcal{A}$; i.e. cluster monomials are linearly independent.

b) Conjecture 7.10 of 21 holds for $\mathcal{A}$; i.e.

1) Different cluster monomials have different $\mathbf{g}$-vectors with respect to a given initial seed.

2) The $\mathbf{g}$-vectors of the cluster variables in any given cluster form a $\mathbb{Z}$-basis of the lattice $\mathbb{Z}^{r}$.

c) Conjecture 7.12 of [21] holds for $\mathcal{A}$; i.e. if $\left(g_{1}, \ldots, g_{r}\right)$ and $\left(g_{1}^{\prime}, \ldots, g_{r}^{\prime}\right)$ are the $\mathbf{g}$-vectors of one and the same cluster variable with respect to two clusters $t$ and $t^{\prime}$ related by the mutation at $l$, then we have

$$
g_{j}^{\prime}= \begin{cases}-g_{l} & \text { if } j=l, \\ g_{j}+\left[b_{j l}\right]_{+} g_{l}-b_{j l} \min \left(g_{l}, 0\right) & \text { if } j \neq l,\end{cases}
$$

where the $b_{i j}$ are the entries of the $r \times r$ matrix $B$ associated with $t$ and we write $[x]_{+}$for $\max (x, 0)$ for any integer $x$.

Proof. a) By Theorem 5.4 a), each cluster monomial $m$ is the image $X_{M}^{\prime}$ of a rigid object $M$ of $\mathcal{E}$, where $M$ does not have any nonzero projective direct factor. Moreover, such an object $M$ is unique up to isomorphism. Thus, given a set $m_{1}$, $\ldots, m_{N}$ of pairwise distinct cluster monomials, we obtain a set $M_{1}, \ldots, M_{N}$ of pairwise nonisomorphic rigid objects without projective direct factors such that $X_{M_{i}}^{\prime}=m_{i}$ for $1 \leq i \leq N$. Thus, by Corollary $4.4 \mathrm{~b}$ ), the images $X_{M_{i}}^{\prime}=m_{i}$ of the $M_{i}$ are not only pairwise distinct but in fact linearly independent.

b) Let us prove 1). Let $m$ and $m^{\prime}$ be two distinct cluster monomials. We would like to compare their $\mathbf{g}$-vectors with respect to a given initial cluster. By Theorem $5.4 \mathrm{a}$ ), we may assume that this given cluster consists of the images under $M \mapsto X_{M}^{\prime}$ of the indecomposable direct factors of $T$. Still by Theorem 5.4 a), the monomials $m$ and $m^{\prime}$ are the images $X_{M}^{\prime}$ and $X_{M^{\prime}}^{\prime}$ of two nonisomorphic rigid objects $M$ and $M^{\prime}$ of $\mathcal{E}$ without nonzero projective direct factors. Thus $M$ and $M^{\prime}$ are still nonisomorphic in the stable category $\mathcal{C}=\underline{\mathcal{E}}$. But by Theorem 2.3 of [13, nonisomorphic rigid objects have distinct indices $\operatorname{ind}_{T}(M)$ and $\operatorname{ind}_{T}\left(M^{\prime}\right)$. Therefore, they have distinct g-vectors by Proposition 4.3. Now let us prove 2). Let a cluster $\mathrm{x}^{\prime}$ be given. By Theorem $5.4 \mathrm{a}$ ), the variables $x_{i}^{\prime}$ in $\mathrm{x}^{\prime}$ are the images under $M \mapsto X_{M}^{\prime}$ of the indecomposable nonprojective direct summands $T_{i}^{\prime}$ of a cluster-tilting object $T^{\prime}$ reachable from $T$. By Proposition 4.3, the g-vector of each $x_{i}^{\prime}$ is the index of $T_{i}^{\prime}$. Now by Theorem 2.6 of [13], the indices of the indecomposable direct factors of a cluster-tilting object form a basis of the lattice $K_{0}(\operatorname{add} \underline{T})$, where $\underline{T}$ is the image of $T$ in $\mathcal{C}$. Thus the $\mathrm{g}$-vectors of the $x_{i}^{\prime}$ form a basis of the lattice $\mathbb{Z}^{r}$. 
c) By Theorem 5.4 a), we may assume that under the maps $M \mapsto X_{M}^{\prime}$, the clusters $t$ and $t^{\prime}$ correspond to the cluster-tilting object $T$ and another cluster-tilting object $T^{\prime}$ obtained from $T$ by mutation at the nonprojective indecomposable direct factor $T_{l}$. Moreover, the given cluster variable $x$ corresponds to some nonprojective rigid indecomposable object $X$. By Proposition 4.3, the g-vectors of $x$ with respect to $t$ and $t^{\prime}$ are given by the components of the indices $\operatorname{ind}_{T}(X)$ and $\operatorname{ind}_{T^{\prime}}(X)$ in the bases formed by the $\operatorname{ind}_{T}\left(T_{i}\right), 1 \leq i \leq r$, respectively the $\operatorname{ind}_{T^{\prime}}\left(T_{i}^{\prime}\right), 1 \leq i \leq r$, where the $T_{i}$ and the $T_{i}^{\prime}$ are the nonprojective indecomposable direct factors of $T$, respectively $T^{\prime}$. Now Theorem 3.1 of [13] tells us exactly how $\operatorname{ind}_{T}(X)$ and ind $_{T^{\prime}}(X)$ are related: Let

$$
T_{l} \longrightarrow E^{\prime} \longrightarrow T_{l}^{*} \longrightarrow \Sigma T_{l} \text { and } T_{l}^{*} \longrightarrow E \longrightarrow T_{l} \longrightarrow \Sigma T_{l}^{*}
$$

be the exchange triangles associated with the mutation from $T$ to $T^{\prime}$. Let

$$
\phi_{+}: K_{0}(\operatorname{add} T) \rightarrow K_{0}\left(\operatorname{add} T^{\prime}\right) \text { and } \phi_{-}: K_{0}(\operatorname{add} T) \rightarrow K_{0}\left(\operatorname{add} T^{\prime}\right)
$$

be the linear maps which send the classes $\left[T_{i}\right], i \neq l$, to themselves and send $\left[T_{l}\right]$ to

$$
\phi_{+}\left(\left[T_{l}\right]\right)=[E]-\left[T_{l}^{*}\right], \text { respectively } \phi_{-}\left(\left[T_{l}\right]\right)=\left[E^{\prime}\right]-\left[T_{l}^{*}\right] .
$$

Then by Theorem 3.1 of 13 , we have

$$
\operatorname{ind}_{T^{\prime}}(X)= \begin{cases}\phi_{+}\left(\operatorname{ind}_{T}(X)\right) & \text { if }\left[\operatorname{ind}_{T}(X): T_{l}\right] \geq 0 \\ \phi_{-}\left(\operatorname{ind}_{T}(X)\right) & \text { if }\left[\operatorname{ind}_{T}(X): T_{l}\right] \leq 0 .\end{cases}
$$

We leave it to the reader to check that this yields exactly the rule given in the assertion.

Let $\tilde{B}$ be a $2 r \times r$ matrix whose principal (i.e. top $r \times r$ ) part $B_{0}$ is mutation equivalent to an acyclic matrix, and whose complementary (i.e. bottom) part is the $r \times r$ identity matrix. Let $\mathcal{A}(\tilde{B})$ be the cluster algebra with the initial seed $(\mathbf{x}, \tilde{B})$.

Theorem 5.6. With the above notation, the cluster algebra $\mathcal{A}(\tilde{B})$ does not admit a Frobenius 2-CY realization.

Proof. Suppose that $\mathcal{A}(\tilde{B})$ has a Frobenius $2-\mathrm{CY}$ realization $\mathcal{E}$. Then there is a cluster-tilting object $T$ of $\mathcal{E}$ with $2 r$ indecomposable direct summands. Then we have $B(T)^{0}=\tilde{B}$. Since $B_{0}$ is mutation equivalent to an acyclic matrix $B_{c}$ by a series of mutations, we have a cluster-tilting object $T^{\prime}$ such that the quiver of the stable endomorphism algebra of $T^{\prime}$ corresponds to $B_{c}$. Let $A$ be the stable endomorphism algebra of $T^{\prime}$. By the main theorem of [33], we have a triangle equivalence $\underline{\mathcal{E}} \simeq \mathcal{C}_{A}$, where $\mathcal{C}_{A}$ is the cluster category of $A$. Thus the cluster-tilting graph of $\mathcal{E}$ is connected and every rigid object of $\mathcal{E}$ can be extended to a clustertilting object of $\mathcal{E}$.

Let $F=\operatorname{Hom}_{\mathcal{E}}(T, ?)$. Let $S_{i}, 1 \leq i \leq 2 r$, be the simple modules of $\operatorname{End}_{\mathcal{E}}(T)$. For each object $M$ of $\mathcal{E}$, we have the Laurent polynomial

$$
X_{M}^{\prime}=\prod_{i=1}^{2 r} x_{i}^{\left\langle F M, S_{i}\right\rangle_{\tau}} \sum_{e} \chi\left(G r_{e}\left(\operatorname{Ext}_{\mathcal{E}}^{1}(T, M)\right)\right) \prod_{i=1}^{2 r} x_{i}^{-\left\langle e, S_{i}\right\rangle_{3}} .
$$

Let

$$
y_{j}=\prod_{i=1}^{2 r} x_{i}^{b_{i j}}, 1 \leq j \leq r .
$$


As in Proposition 4.3, we can rewrite $X_{M}^{\prime}$ as

$$
X_{M}^{\prime}=\prod_{i=1}^{2 r} x_{i}^{\left\langle F M, S_{i}\right\rangle_{\tau}}\left(1+\sum_{e \neq 0} \chi\left(G r_{e}\left(\operatorname{Ext}_{\mathcal{E}}^{1}(T, M)\right)\right) \prod_{i=1}^{r} y_{j}^{e_{j}}\right),
$$

where $e_{j}$ is the $j$-th coordinate of $e$ in the basis of the $S_{i}, 1 \leq i \leq 2 r$. If the indecomposable object $M$ is rigid and not isomorphic to $T_{i}$ for $r<i \leq 2 r$, then $X_{M}^{\prime}$ is a cluster variable of $\mathcal{A}(\tilde{B})$. By the definition of the rational function $\mathcal{F}_{l, t}$ associated with the cluster variable $x_{l, t}$ in 21 , we have

$$
\begin{aligned}
\mathcal{F}_{M} & =X_{M}^{\prime}\left(x_{1}=x_{2}=\ldots=x_{r}=1\right) \\
& =\prod_{i=r+1}^{2 r} x_{i}^{\left\langle F M, S_{i}\right\rangle_{\tau}}\left(1+\sum_{e \neq 0} \chi\left(G r_{e}\left(\operatorname{Ext}_{\mathcal{E}}^{1}(T, M)\right)\right) \prod_{j=r+1}^{2 r} x_{j}^{e_{j-r}}\right) .
\end{aligned}
$$

Put

$$
G_{M}=1+\sum_{e \neq 0} \chi\left(G r_{e} \operatorname{Ext}_{\mathcal{E}}^{1}(T, M)\right) \prod_{j=r+1}^{2 r} x_{j}^{e_{j-r}} .
$$

Note that $G_{M}$ is always a polynomial of $x_{i}, r+1 \leq i \leq 2 r$, with constant term 1. By Proposition 5.2 in [21], we know that the polynomial $\mathcal{F}_{M}$ is not divisible by $x_{i}, r+1 \leq i \leq 2 r$. Now for $i>r$, we have $\left\langle F M, S_{i}\right\rangle_{\tau} \geq 0$ in general, which implies that $\left\langle F M, S_{i}\right\rangle_{\tau}=0$. In particular, $\left\langle F M, S_{i}\right\rangle_{\tau}=\left[\operatorname{ind}_{T}(M): T_{i}\right]=0$, for $r+1 \leq i \leq 2 r$. Consider $M=\Sigma T_{1}$, which is rigid and indecomposable, so $X_{M}^{\prime}$ is a cluster variable of the cluster algebra $\mathcal{A}(\tilde{B})$. But in the Frobenius category $\mathcal{E}$ we have the conflation

$$
0 \rightarrow T_{1} \rightarrow P \rightarrow \Sigma T_{1} \rightarrow 0,
$$

where $P$ is an injective hull of $T_{1}$, which implies

$$
\operatorname{ind}_{T}(M)=[P]-\left[T_{1}\right] .
$$

Thus there is always some $r+1 \leq i \leq 2 r$ such that $\left[\operatorname{ind}_{T}(M): T_{i}\right] \neq 0$, a contradiction.

Remark 5.7. In the above notation, if $B_{0}$ is acyclic, then it is easy to deduce that the cluster algebra $\mathcal{A}(\tilde{B})$ does not have a Frobenius 2-CY realization. Indeed in this case, one of the first $r$ vertices of $Q$ which corresponds to $\tilde{B}$ is always a sink. This is incompatible with the existence of a Frobenius 2-CY realization by Remark 5.2,

\section{Triangulated 2-Calabi-Yau REalizations}

6.1. Definitions. Let $B=\left(b_{i j}\right)_{n \times n}$ be an antisymmetric integer matrix and $\mathcal{A}(B)$ the associated cluster algebra. A 2-Calabi-Yau triangulated category $\mathcal{C}$ is called a triangulated 2-Calabi-Yau realization of the matrix $B$ if $\mathcal{C}$ admits a cluster-tilting object $T$ such that

- $\mathcal{C}$ has a cluster structure in the sense [2]; $f f$. section 2.4,

- $T$ has exactly $n$ nonisomorphic indecomposable direct summands $T_{1}, \ldots$, $T_{n}$. 
- The antisymmetric matrix $B(T)$ associated with the quiver of the endomorphism algebra of $T$ equals $B$.

We denote a triangulated 2-CY realization of $B$ by $\mathcal{C} \supset$ add $T$.

Let $n_{1}$ and $n_{2}$ be positive integers. Let $B_{1}$ and $B_{2}$ be antisymmetric integer $n_{1} \times$ $n_{1}$, resp. $n_{2} \times n_{2}$ matrices. Let $B_{21}$ be an integer $n_{2} \times n_{1}$ matrix with nonnegative entries. Let $\mathcal{C}_{i} \supset \mathcal{T}_{i}$ be a triangulated 2 -CY realization of $B_{i}, i=1,2$. Let $B$ be the matrix

$$
\left(\begin{array}{cc}
B_{1} & -B_{21}^{t} \\
B_{21} & B_{2}
\end{array}\right)
$$

A gluing of $\mathcal{C}_{1} \supset \mathcal{T}_{1}$ with $\mathcal{C}_{2} \supset \mathcal{T}_{2}$ with respect to $B$ is a triangulated 2-CY realization $\mathcal{C} \supset \mathcal{T}$ of $B$ endowed with full additive subcategories $\mathcal{T}_{1}^{\prime}$ and $\mathcal{T}_{2}^{\prime}$ such that

- $\operatorname{Hom}_{\mathcal{C}}\left(\mathcal{T}_{1}^{\prime}, \mathcal{T}_{2}^{\prime}\right)=0$.

- The set indec $(\mathcal{T})$ is the disjoint union of indec $\left(\mathcal{T}_{1}^{\prime}\right)$ with indec $\left(\mathcal{T}_{2}^{\prime}\right)$.

- There is a triangle equivalence

$$
{ }^{\perp}\left(\Sigma \mathcal{T}_{1}^{\prime}\right) /\left(\mathcal{T}_{1}^{\prime}\right) \stackrel{\sim}{\longrightarrow} \mathcal{C}_{2}
$$

inducing an equivalence $\mathcal{T}_{2}^{\prime} \stackrel{\sim}{\longrightarrow} \mathcal{T}_{2}$.

- There is a triangle equivalence

$$
{ }^{\perp}\left(\Sigma \mathcal{T}_{2}^{\prime}\right) /\left(\mathcal{T}_{2}^{\prime}\right) \stackrel{\sim}{\longrightarrow} \mathcal{C}_{1}
$$

inducing an equivalence $\mathcal{T}_{1}^{\prime} \stackrel{\sim}{\longrightarrow} \mathcal{T}_{1}$.

A principal gluing of $\mathcal{C}_{1} \supset \mathcal{T}_{1}$ is a gluing of $\mathcal{C}_{1} \supset \mathcal{T}_{1}$ with $\mathcal{C}_{2} \supset \mathcal{T}_{2}$ with respect to

$$
\left(\begin{array}{cc}
B_{1} & -I_{n_{1}} \\
I_{n_{1}} & 0
\end{array}\right)
$$

where $\mathcal{C}_{2}$ is the cluster category of $\left(A_{1}\right)^{n_{1}}$ and $\mathcal{T}_{2}$ is the image of the subcategory of finitely generated projective modules.

It is well known that each acyclic matrix $B$ admits a triangulated 2-CY realization $\mathcal{C}_{Q_{B}}$, where $\mathcal{C}_{Q_{B}}$ is the cluster category of the quiver $Q_{B}$ corresponding to $B$. In the last subsection, we will see that $\mathcal{C}_{Q_{B}}$ does admit a principal gluing.

Conjecture 6.1. If $\mathcal{C}_{1}$ and $\mathcal{C}_{2}$ are algebraic, a gluing exists for any matrix $B_{21}$ with nonnegative entries.

Amiot's work [1] provides some evidence for the conjecture. Indeed, if $\mathcal{C}_{1}$ and $\mathcal{C}_{2}$ are generalized cluster categories [1] associated with Jacobi-finite quivers with potential [15, it is easy to construct a quiver with potential which provides a gluing as required by the conjecture.

6.2. Cluster algebras with coefficients. Let $B$ be an antisymmetric integer $n \times n$ matrix. Suppose that the matrix $B$ admits a triangulated 2 -CY realization $\mathcal{C}$ with the cluster-tilting subcategory $\mathcal{T}=\operatorname{add} T$. Let $T_{i}, 1 \leq i \leq n$, be the nonisomorphic indecomposable direct summands of $T$. By the definition, we have $B(T)=B$. The mutations of the matrix $B$ correspond to the mutations of the cluster-tilting object $T$. Fix an integer $0<r \leq n$ and consider the submatrix $B^{0}$ of $B$ formed by the first $r$ columns of $B$. If $l \leq r$, then we have

$$
\mu_{l}\left(B^{0}\right)=\left(\mu_{l}(B)\right)^{0},
$$


where $\mu_{l}$ is the mutation in the direction $l$. Thus we can view the cluster algebra $\mathcal{A}\left(B^{0}\right)$ with coefficients as a subcluster algebra of $\mathcal{A}(B) ; c f$. Ch. III of 2 .

Denote by $\mathcal{P}$ the full subcategory of $\mathcal{C}$ whose objects are the finite direct sums of copies of $T_{r+1}, \ldots, T_{n}$. We define a subcategory of $\mathcal{C}$ as follows:

$$
\mathcal{U}={ }^{\perp}(\Sigma \mathcal{P})=\left\{X \in \mathcal{C} \mid \operatorname{Ext}_{\mathcal{C}}^{1}\left(T_{i}, X\right)=0 \text { for } r<i \leq n\right\} .
$$

By Theorem I.2.1 of [2], the quotient category $\mathcal{U} / \mathcal{P}$ is a 2-Calabi-Yau triangulated category and the projection $\mathcal{U} \rightarrow \mathcal{U} / \mathcal{P}$ induces a bijection between the clustertilting subcategories of $\mathcal{C}$ containing $\mathcal{P}$ and the cluster-tilting subcategories of $\mathcal{U} / \mathcal{P}$. Thus, a mutation of a cluster-tilting object in $\mathcal{U} / \mathcal{P}$ can be viewed as a mutation of a cluster-tilting object in $\mathcal{U} \subset \mathcal{C}$ which does not affect the direct summands $T_{i}$, $r<i \leq n$. This exactly corresponds to a mutation of the matrix $B$ in one of the first $r$ directions. In particular, a mutation of the cluster algebra $\mathcal{A}\left(B^{0}\right)$ corresponds to a mutation of a cluster-tilting object in $\mathcal{U}$.

Recall from section 2.5 that on $\mathcal{C}$, we have Palu's cluster character associated with $T$, which is given by the formula

$$
X_{M}=X_{M}^{T}=\prod_{i=1}^{n} x_{i}^{-\left[\operatorname{coind}_{T} M: T_{i}\right]} \sum_{e} \chi\left(G r_{e}\left(\operatorname{Hom}_{\mathcal{C}}(T, M)\right)\right) \prod_{i=1}^{n} x_{i}^{\left\langle S_{i}, e\right\rangle_{a}} .
$$

We consider the composition of this map with the shift:

$$
X_{M}^{\prime}=X_{\Sigma M}=\prod_{i=1}^{n} x_{i}^{\left[\operatorname{ind}_{T} M: T_{i}\right]} \sum_{e} \chi\left(G r_{e}\left(\operatorname{Hom}_{\mathcal{C}}(T, \Sigma M)\right)\right) \prod_{i=1}^{n} x_{i}^{\left\langle S_{i}, e\right\rangle_{a}} .
$$

We consider the restriction of the map $M \mapsto X_{M}^{\prime}$ to the subcategory $\mathcal{U}$. It follows from Proposition 2.3 that if $M$ is an indecomposable rigid object reachable from $T$ in $\mathcal{U}$, then $X_{M}^{\prime}$ is a cluster variable of $\mathcal{A}\left(B^{0}\right)$. We will rewrite this variable so as to express its g-vector (if it is defined) in terms of the index of $M$ : Let $M$ be an object of $\mathcal{U}$. Then $\operatorname{Hom}_{\mathcal{C}}(T, \Sigma M)$ is an $\operatorname{End}_{\mathcal{C}}(T)$-module which vanishes at each vertex $r<i \leq n$. Let $e$ be the image of $\operatorname{Hom}_{\mathcal{C}}(T, \Sigma M)$ in the Grothendieck group of $\bmod \operatorname{End}_{\mathcal{C}}(T)$. Let $e_{j}$ be the $j$-th coordinate of $e$ with respect to the basis $S_{i}$, $1 \leq i \leq n$. We have

$$
\begin{aligned}
\left\langle S_{i}, e\right\rangle_{a} & =\left\langle S_{i}, e\right\rangle_{\tau}-\left\langle e, S_{i}\right\rangle_{\tau} \\
& =\sum_{j=1}^{r} e_{j}\left(\left\langle S_{i}, S_{j}\right\rangle_{\tau}-\left\langle S_{j}, S_{i}\right\rangle_{\tau}\right) \\
& =\sum_{j=1}^{r} e_{j}\left(\operatorname{Ext}_{\operatorname{End}_{\mathcal{C}}(T)}^{1}\left(S_{j}, S_{i}\right)-\operatorname{Ext}_{\operatorname{End}_{\mathcal{C}}(T)}^{1}\left(S_{i}, S_{j}\right)\right) \\
& =\sum_{j=1}^{r} b_{i j} e_{j} .
\end{aligned}
$$

As in section 4, put

$$
y_{j}=\prod_{i=1}^{n} x_{i}^{b_{i j}}, \text { for } 1 \leq j \leq r
$$

Then $X_{M}^{\prime}$ can be rewritten as

$$
X_{M}^{\prime}=\prod_{i=1}^{n} x_{i}^{\left[\operatorname{ind}_{T}(M): T_{i}\right]}\left(1+\sum_{e \neq 0} \chi\left(G r_{e}\left(\operatorname{Hom}_{\mathcal{C}}(T, \Sigma M)\right)\right) \prod_{j=1}^{r} y_{j}^{e_{j}}\right) .
$$


As in section 4, when rank $B^{0}=r$, we can define the g-vector of $M \in \mathcal{U}$ with respect to a cluster tilting object $T$. Thus we have proved part a) of the following proposition. We leave the easy proof of part b) to the reader.

Proposition 6.2. Suppose that rank $B^{0}=r$. Let $M$ be an object of $\mathcal{U}$.

a) The $\mathbf{g}$-vector of $X_{M}^{\prime}$ with respect to the initial cluster is given by

$$
g_{T}\left(X_{M}^{\prime}\right)(i)=\left[\operatorname{ind}_{T}(M): T_{i}\right], \text { for } 1 \leq i \leq r .
$$

b) The index of the image of $M$ in $\mathcal{U} / \mathcal{P}$ with respect to the image of $T$ is

$$
\sum_{i=1}^{r} g_{T}\left(X_{M}^{\prime}\right)(i)\left[T_{i}\right]
$$

In analogy with the definition in section 5 , we define a cluster-tilting object $T^{\prime}$ of $\mathcal{U}$ to be reachable from $T$ if it is obtained from $T$ by a sequence of mutations at indecomposable rigid objects of $\mathcal{U}$ not in $\mathcal{P}$. We define an indecomposable rigid object of $\mathcal{U}$ to be reachable from $T$ if it is a direct factor of a cluster-tilting object reachable from $T$.

Theorem 6.3. Let $B$ be an antisymmetric integer $n \times n$ matrix and $1 \leq r \leq n$ an integer such that the submatrix $B^{0}$ of $B$ formed by the first $r$ columns has rank $r$. Let $\mathcal{A}=\mathcal{A}\left(B^{0}\right)$ be the associated cluster algebra with coefficients. Assume that the matrix $B$ admits a triangulated 2-CY realization given by a triangulated category $\mathcal{C}$ with a cluster tilting object $T$ which is the sum of $n$ indecomposable direct factors $T_{1}, \ldots, T_{n}$. Denote by $\mathcal{P}$ the full subcategory of $\mathcal{C}$ whose objects are the finite direct sums of copies of $T_{r+1}, \ldots, T_{n}$ and define the subcategory $\mathcal{U}$ of $\mathcal{C}$ by

$$
\mathcal{U}={ }^{\perp}(\Sigma \mathcal{P})=\left\{X \in \mathcal{C} \mid \operatorname{Ext}_{\mathcal{C}}^{1}\left(T_{i}, X\right)=0 \text { for } r<i \leq n\right\} .
$$

For $M \in \mathcal{C}$, define (cf. section 2.5)

$$
X_{M}^{\prime}=X_{\Sigma M}^{T}=\prod_{i=1}^{n} x_{i}^{\left[\operatorname{ind}_{T} M: T_{i}\right]} \sum_{e} \chi\left(G r_{e}\left(\operatorname{Hom}_{\mathcal{C}}(T, \Sigma M)\right)\right) \prod_{i=1}^{n} x_{i}^{\left\langle S_{i}, e\right\rangle_{a}} .
$$

Then the following hold.

a) The map $M \mapsto X_{M}^{\prime}$ induces a bijection from the set of isomorphism classes of indecomposable rigid objects of $\mathcal{U}$ not belonging to $\mathcal{P}$ and reachable from $T$ onto the set of cluster variables of $\mathcal{A}\left(B^{0}\right)$. Under this bijection, the cluster-tilting objects of $\mathcal{U}$ reachable from $T$ correspond to the clusters of $\mathcal{A}\left(B^{0}\right)$.

b) The map $M \mapsto\left[\operatorname{ind}_{T}(M): T_{i}\right]_{1 \leq i \leq r}$ is a bijection from the set of indecomposable rigid objects of $\mathcal{U}$ not belonging to $\mathcal{P}$ and reachable from $T$ onto the set of $\mathbf{g}$-vectors of cluster variables of $\mathcal{A}\left(B^{0}\right)$.

c) Conjecture 7.2 of [21] holds for $\mathcal{A}$; i.e. the cluster monomials are linearly independent over $\mathbb{Z}$. Moreover, the cluster monomials form a basis of the $\mathbb{Z}\left[x_{r+1}, \ldots, x_{n}\right]$-submodule of $\mathcal{A}\left(B^{0}\right)$ which they generate.

d) Conjecture 7.10 of 21 holds for $\mathcal{A}$; i.e.

1) Different cluster monomials have different $\mathbf{g}$-vectors with respect to a given initial seed.

2) The $\mathbf{g}$-vectors of the cluster variables in any given cluster form a $\mathbb{Z}$-basis of the lattice $\mathbb{Z}^{r}$. 
e) Conjecture 7.12 of [21] holds for $\mathcal{A}$; i.e. if $\left(g_{1}, \ldots, g_{r}\right)$ and $\left(g_{1}^{\prime}, \ldots, g_{r}^{\prime}\right)$ are the $\mathbf{g}$-vectors of one and the same cluster variable with respect to two clusters $t$ and $t^{\prime}$ related by the mutation at $l$, then we have

$$
g_{j}^{\prime}=\left\{\begin{array}{lc}
-g_{l} & \text { if } j=l, \\
g_{j}+\left[b_{j l}\right]_{+} g_{l}-b_{j l} \min \left(g_{l}, 0\right) & \text { if } j \neq l,
\end{array}\right.
$$

where the $b_{i j}$ are the entries of the $r \times r$ matrix $B$ associated with $t$ and we write $[x]_{+}$for $\max (x, 0)$ for any integer $x$.

Proof. It follows from Proposition 2.3 that the map $M \mapsto X_{M}^{\prime}$ is well-defined and surjective onto the set of cluster variables of $\mathcal{A}\left(B^{0}\right)$. It is injective by Proposition $6.2 \mathrm{~b})$ because rigid objects of $\mathcal{U} / \mathcal{P}$ are determined by their indices and the map taking a rigid object $M$ of $\mathcal{U}$ without nonzero direct factors in $\mathcal{P}$ to its image in $\mathcal{U} / \mathcal{P}$ is injective (up to isomorphism). This also implies part b). The same proof as for Corollary $4.4 \mathrm{~b}$ ) yields the linear independence of the cluster monomials in c). Let us prove that the cluster monomials form a basis of the $\mathbb{Z}\left[x_{r+1}, \ldots, x_{n}\right]$-submodule of $\mathcal{A}\left(B^{0}\right)$ which they generate. Indeed, over $\mathbb{Z}$, this submodule is spanned by the images $X_{M}^{\prime}$ of all rigid objects of $\mathcal{U}$ obtained as direct sums of objects of $\mathcal{P}$ and indecomposable rigid objects reachable from $T$ not belonging to $\mathcal{P}$. Such objects $M$ are, in particular, rigid in $\mathcal{T}$ and they can be distinguished (up to isomorphism) by their indices. Now again, the same proof as for Corollary $4.4 \mathrm{~b}$ ) shows that these $X_{M}^{\prime}$ are linearly independent over $\mathbb{Z}$. Clearly this implies that the cluster monomials form a basis of the $\mathbb{Z}\left[x_{r+1}, \ldots, x_{n}\right]$-submodule of $\mathcal{A}\left(B^{0}\right)$ which they generate. As in the proof of Theorem [5.5 b), the assertions in part d) follow from the interpretation of the $\mathbf{g}$-vector given in $6.2 \mathrm{~b}$ ) and the facts that

1) rigid objects of $\mathcal{U} / \mathcal{P}$ are determined by their indices (Theorem 2.3 of [13]) and

2) the indices of the indecomposable direct factors of a cluster-tilting subcategory $\mathcal{T}$ of $\mathcal{U} / \mathcal{P}$ form a basis of $K_{0}(\mathcal{T})$ (Theorem 2.6 of [13]).

Part e) is proved in exactly the same way as the corresponding statement for cluster algebras with a 2-CY Frobenius realization in Theorem $5.4 \mathrm{c}$ ).

Example 6.4. Let $A_{4}$ be the quiver $3 \rightarrow 1 \rightarrow 2 \leftarrow 4, \mathcal{C}_{Q}$ the corresponding cluster category. The following is the AR quiver of $\mathcal{C}_{Q}$, where $P_{i}, 1 \leq i \leq 4$, are the indecomposable projective $k Q$-modules.

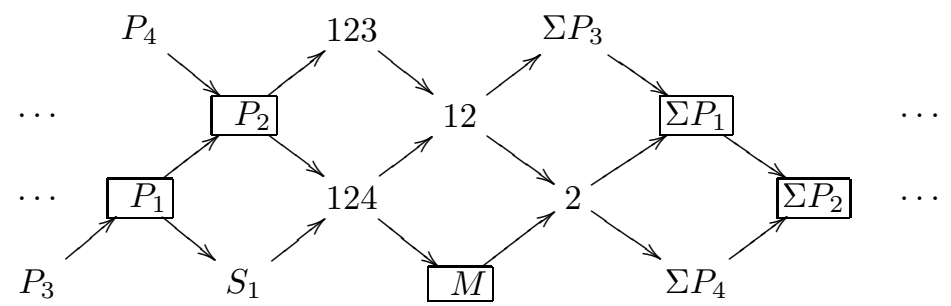

Let $T=P_{1} \oplus P_{2} \oplus P_{3} \oplus P_{4}$ be the canonical cluster-tilting object in $\mathcal{C}_{Q}, \mathcal{P}=$ $\operatorname{add}\left(P_{3} \oplus P_{4}\right)$. It is easy to see that the indecomposable objects in $\mathcal{U} / \mathcal{P} \cong \mathcal{C}_{A_{2}}$ are 
exactly $P_{1}, P_{2}, M, \Sigma P_{1}, \Sigma P_{2}$. In this case, the matrix $B(T)^{0}$ is

$$
\left(\begin{array}{ll}
0 & 1 \\
-1 & 0 \\
1 & 0 \\
0 & 1
\end{array}\right)
$$

We have rank $B(T)^{0}=2$. Moreover, the cluster algebra $\mathcal{A}\left(B(T)^{0}\right)$ has principal coefficients.

6.3. Cluster algebras with principal coefficients. In this subsection, we suppose that $2 r=n$ and that the complementary part of $B^{0}$ is the $r \times r$ identity matrix. Thus the cluster algebra $\mathcal{A}\left(B^{0}\right)$ has principal coefficients. Recall that for the matrix $B$, we have a triangulated 2 -CY realization $\mathcal{C} \supset$ add $T$ and we have fixed $\mathcal{P}=\operatorname{add}\left(T_{r+1} \oplus \ldots \oplus T_{2 r}\right)$. Let $\mathcal{Q}=\operatorname{add}\left(T_{1} \oplus \ldots \oplus T_{r}\right)$. Let $\mathcal{C}_{1}=\mathcal{U} / \mathcal{P}$ and $\mathcal{C}_{2}={ }^{\perp}(\Sigma \mathcal{Q}) / \mathcal{Q}$ be the quotient categories, $\mathcal{T}_{1}=\operatorname{add}\left(\pi_{1}\left(T_{1} \oplus \ldots \oplus T_{r}\right)\right)$ and $\mathcal{T}_{2}=\operatorname{add}\left(\pi_{2}\left(T_{r+1} \oplus \ldots \oplus T_{2 r}\right)\right)$ the corresponding cluster-tilting subcategories, where $\pi_{1}$ and $\pi_{2}$ are the respective projection functors. Then $\mathcal{C}$ is a gluing of $\mathcal{C}_{1} \supset \mathcal{T}_{1}$ with $\mathcal{C}_{2} \supset \mathcal{T}_{2}$ with respect to the matrix $B$.

As in section 5, for a cluster variable $x_{l, t}$ of the cluster algebra $\mathcal{A}\left(B^{0}\right)$ which corresponds to an indecomposable rigid object $M \in \mathcal{U}$ and not in $\mathcal{P}$, we denote the rational function $\mathcal{F}_{l, t}$ defined in section 3 of [21] by $\mathcal{F}_{M}$. Since $x_{l, t}=X_{M}^{\prime}$, we have

$$
\begin{aligned}
\mathcal{F}_{M} & =X_{M}^{\prime}\left(x_{1}=\ldots=x_{r}=1\right) \\
& =\prod_{i=r+1}^{2 r} x_{i}^{\left[\operatorname{ind}_{T}(M): T_{i}\right]}\left(1+\sum_{e \neq 0} \chi\left(G r_{e}\left(\operatorname{Hom}_{\mathcal{C}}(T, \Sigma M)\right)\right) \prod_{j=r+1}^{2 r} x_{j}^{e_{j-r}}\right) .
\end{aligned}
$$

The following result is now a consequence of Proposition 3.6 and 5.2 in 21. We give a proof based on representation theory. Note that Conjecture 5.4 of [21] will be proved in full generality in [16].

Theorem 6.5. Conjecture 5.4 of 21 holds for $\mathcal{A}\left(B^{0}\right)$; i.e. the polynomial $\mathcal{F}_{M}$ has constant term 1 . Thus we have

$$
\mathcal{F}_{M}=1+\sum_{e \neq 0} \chi\left(G_{e}\left(\operatorname{Hom}_{\mathcal{C}}(T, \Sigma M)\right)\right) \prod_{j=r+1}^{2 r} x_{j}^{e_{j-r}} .
$$

Proof. We need to show that for each $i>r$, $\left[\operatorname{ind}_{T}(M): T_{i}\right]$ is zero. Since $X_{M}^{\prime}$ is a cluster variable and $M$ is indecomposable, we have the following two cases:

Case 1: $M \cong \Sigma T_{j}$ for some $j \leq r$. We have $\operatorname{ind}_{T}(M)=-\left[T_{j}\right]$, which implies that $\left[\operatorname{ind}_{T}(M): T_{i}\right]=0$.

Case 2: $M$ is not isomorphic to $\Sigma T_{j}$ for any $j \leq r$. Recall that by assumption, $M$ is not isomorphic to $T_{j}$ for any $j>r$. We have the following minimal triangle:

$$
T_{M}^{1} \rightarrow T_{M}^{0} \rightarrow M \rightarrow \Sigma T_{M}^{1}
$$

with $T_{M}^{0}, T_{M}^{1}$ in add $T$ and $\operatorname{ind}_{T}(M)=\left[T_{M}^{0}\right]-\left[T_{M}^{1}\right]$. Since $M$ belongs to $\mathcal{U}$, for each $i>r$ we have $\operatorname{Hom}_{\mathcal{C}}\left(M, \Sigma T_{i}\right)=0$. If we had $\left[T_{M}^{1}: T_{i}\right] \neq 0$ for some $i>r$, then the above minimal triangle would have a nonzero direct factor

$$
T_{i} \rightarrow T_{i} \rightarrow 0 \rightarrow \Sigma T_{i} .
$$

Suppose that we have $\left[T_{M}^{0}: T_{i}\right] \neq 0$ for some $i>r$. Applying the functor $F=$ $\operatorname{Hom}_{\mathcal{C}}(T, ?)$ to the triangle, we get a minimal projective resolution of $F M$ as an 
$\operatorname{End}_{\mathcal{C}}(T)$-module. Note that for $i>r$, the projective module $F T_{i}$ is also a simple module, which implies that $F M$ is decomposable, a contradiction.

Suppose that the indecomposable rigid object $M$ of $\mathcal{C}$ is reachable from $T$ and consider the polynomial $\mathcal{F}_{M}$ of Theorem 6.5. We define the $f$-vector $f_{T}(M)=$ $\left(f_{1}, \ldots, f_{r}\right)$ of $M$ with respect to $T$ by

$$
\left.\mathcal{F}_{M}\right|_{\text {Trop }\left(u_{1}, \ldots, u_{r}\right)}\left(u_{1}^{-1}, \ldots, u_{r}^{-1}\right)=u_{1}^{-f_{1}} \ldots u_{r}^{-f_{r}},
$$

where $\operatorname{Trop}\left(u_{1}, \ldots, u_{r}\right)$ is the tropical semifield defined in section 2.1 .

Proposition 6.6. Suppose that $M$ is not isomorphic to $T_{i}$ for $1 \leq i \leq 2 r$, and let $\underline{\operatorname{dim}} \operatorname{Hom}_{\mathcal{C}}(T, \Sigma M)=\left(d_{1}, \ldots, d_{r}\right)$. Then we have

$$
d_{i}=f_{i}, 1 \leq i \leq r
$$

Proof. By Theorem 6.5, we have

$$
\mathcal{F}_{M}=1+\sum_{e \neq 0} \chi\left(G r_{e}\left(\operatorname{Hom}_{\mathcal{C}}(T, \Sigma M)\right)\right) \prod_{j=r+1}^{2 r} x_{j}^{e_{j-r}} .
$$

Therefore, we obtain

$$
\begin{aligned}
\left.\mathcal{F}_{M}\right|_{\operatorname{Trop}\left(u_{1}, \ldots, u_{r}\right)}\left(u_{1}^{-1}, \ldots, u_{r}^{-1}\right) & =1 \oplus \bigoplus_{e \neq 0} \chi\left(G r_{e}\left(\operatorname{Hom}_{\mathcal{C}}(T, \Sigma M)\right)\right) \prod_{j=1}^{r} u_{j}^{-e_{j}} \\
& =u_{1}^{-d_{1}} \ldots u_{n}^{-d_{r}} .
\end{aligned}
$$

Under the assumptions above, we have proved that the dimension vector of $\operatorname{Hom}_{\mathcal{C}}(T, \Sigma M)$ equals the $f$-vector $f_{T}(M)$. Conjecture 7.17 of [21] states that the $f$-vectors coincide with the denominator vectors in general. But by recent work of A. Buan, R. Marsh and I. Reiten [5, the dimension vectors do not always coincide with the denominator vectors. In fact, as shown in [5], for a quiver $Q$ whose underlying graph is an affine Dynkin diagram, the vector $\operatorname{dim} \operatorname{Hom}_{\mathcal{C}_{Q}}(T, M)$ is different from the denominator vector of $X_{M}^{T}$ if $M=R$ and $R$ is a direct factor of $T$, where $R$ is a rigid regular indecomposable of maximal quasi-length. This leads to the following minimal counterexample to Conjecture 7.17 in [21. Let us point out that the corresponding computations already appear in 12. In subsection 5.5 below, we will show that in many cases, the $f$-vector is greater than or equal to the denominator vector.

\subsection{A counterexample.}

Example 6.7. Let $Q$ be the following quiver:

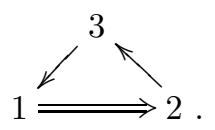

Let $\mathcal{A}(Q)$ be the cluster algebra associated with the initial seed given by $Q$ and $\mathbf{x}=\left(x_{1}, x_{2}, x_{3}\right)$. Consider the mutations at $3,2,1$. Let $\mathbf{x}^{t_{3}}$ be the corresponding cluster. We have

$$
x_{1}^{t_{3}}=\frac{x_{1}^{2}+2 x_{1} x_{2}+x_{2}^{2}+x_{3}}{x_{1} x_{2} x_{3}},
$$


and the corresponding $F$-polynomial is

$$
F_{x_{1}^{t_{3}}}=1+\left(1+y_{1}+y_{1} y_{2}\right) y_{3}+y_{1} y_{2} y_{3}^{2}
$$

Then the $f$-vector of $x_{1}^{t_{3}}$ does not coincide with the denominator vector.

Let us interpret this counterexample in terms of representation theory. Let $A_{2,1}$ be the quiver

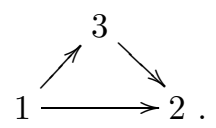

Consider the cluster category $\mathcal{C}_{A_{2,1}}$ of $k A_{2,1}$. Let $P_{i}, 1 \leq i \leq 3$, be the indecomposable projective modules and $S_{i}$ the corresponding simple modules. Then

$$
T=P_{1} \oplus P_{2} \oplus \tau S_{3}
$$

is a cluster-tilting object of $\mathcal{C}_{A_{2,1}}$, where $\tau$ is the Auslander-Reiten translation functor. The quiver $Q_{T}$ of $T$ looks like

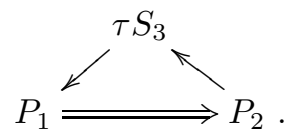

We will show that the cluster category $\mathcal{C}_{A_{2,1}} \supset$ add $T$ admits a principal gluing. For this, consider the following quiver $Q_{1}$ :<smiles>[As][Te]12SOP1[IH]2</smiles>

It admits a cluster category $\mathcal{C}_{Q_{1}}$. Let $T_{Q_{1}}=k Q_{1}$ be the canonical cluster-tilting object in $\mathcal{C}_{Q_{1}}$. Let $T^{\prime}=\mu_{3}\left(\mu_{6}\left(T_{Q_{1}}\right)\right)$ be the cluster-tilting object obtained by mutations from $T_{Q_{1}}$. Denote the nonisomorphic indecomposable direct summands of $T^{\prime}$ by $T_{i}^{\prime}, 1 \leq i \leq 6$. Then the quiver of $Q_{T^{\prime}}$ is

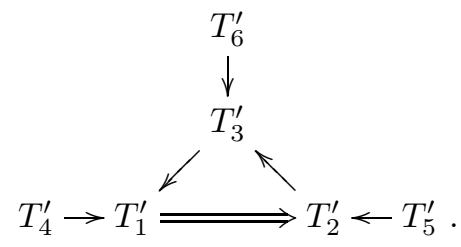

Let $\mathcal{P}=\operatorname{add}\left(T_{4}^{\prime} \oplus T_{5}^{\prime} \oplus T_{6}^{\prime}\right)$. Then $\mathcal{U} / \mathcal{P}$ is a 2-Calabi-Yau triangulated category and admits a cluster-tilting object with the quiver $Q_{T}$. By the main theorem of [33], we know that there is a triangle equivalence $\mathcal{U} / \mathcal{P} \simeq \mathcal{C}_{A_{2,1}}$. Thus, we see that the matrix $B\left(T^{\prime}\right)$ admits a triangulated $2-\mathrm{CY}$ realization $\mathcal{C}_{Q_{1}}$ which is the required principal gluing of $\mathcal{C}_{A_{2,1}} \supset$ add $T$. We may assume that the images of $T_{1}^{\prime}, T_{2}^{\prime}, T_{3}^{\prime}$ coincide with $P_{1}, P_{2}, \tau S_{3}$ in $\mathcal{C}_{A_{2,1}}$ respectively. Denote the shift functor in $\mathcal{C}_{Q_{1}}$ (resp. $\mathcal{C}_{A_{2,1}}$ ) by $\Sigma$ (resp. [1]).

Let $N$ be the preimage of $S_{3}$ in $\mathcal{C}_{Q_{1}}$. Then one can easily compute

$$
\underline{\operatorname{dim}} \operatorname{Hom}_{\mathcal{C}_{Q_{1}}}\left(T^{\prime}, \Sigma N\right)=\underline{\operatorname{dim}} \operatorname{Hom}_{\mathcal{C}_{A_{2,1}}}\left(T, \tau S_{3}\right)=(1,1,2) .
$$

Note that the denominator vector of $X_{N}^{\prime}$ equals the denominator vector of $X_{\tau S_{3}}^{T}$. Now the result follows from the proposition above. 
6.5. An inequality. Let $\mathcal{T}$ be a 2-Calabi-Yau triangulated category with clustertilting object $T$. Recall that we have the generalized Caldero-Chapoton map

$$
X_{M}^{T}=\prod_{i=1}^{n} x_{i}^{-\left[\operatorname{coind}_{T}(M): T_{i}\right]} \sum_{e} \chi\left(G r_{e}(G M)\right) \prod_{i=1}^{n} x_{i}^{\left\langle S_{i}, e\right\rangle_{a}},
$$

where $G$ is the functor $\operatorname{Hom}_{\mathcal{C}}(T, ?): \mathcal{C} \rightarrow \bmod _{\operatorname{End}}(T)$. The following proposition is proved in greater generality in [16].

Proposition 6.8. For each $M$ in $\mathcal{T}$, let $\underline{\operatorname{dim}} G M=\left(m_{1}, \ldots, m_{n}\right)$ and let $1 \leq i \leq$ n. We have

$$
-\left[\operatorname{coind}_{T}(M): T_{i}\right]+\left\langle S_{i}, e\right\rangle_{a} \geq-m_{i}
$$

for each submodule $N$ of $G M$ with $\underline{\operatorname{dim}} N=e$. Thus the exponent of $x_{i}$ in the denominator of $X_{M}$ is less than or equal to $m_{i}$.

Proof. This result holds for the case $M \cong \Sigma T^{\prime}, T^{\prime} \in$ add $T$ obviously. We assume that $M$ is indecomposable and not isomorphic to any $\Sigma T^{\prime}$. The case where $M$ is decomposable is a consequence of the multiplication theorem for $X_{\text {? }}$. Now by Lemma 7 of [37, we have

$$
-\left[\operatorname{coind}_{T}(M): T_{i}\right]=-\left\langle S_{i}, G M\right\rangle_{\tau} .
$$

Note that we have the short exact sequence of $\operatorname{End}_{\mathcal{C}}(T)$-modules

$$
0 \rightarrow N \rightarrow G M \rightarrow G M / N \rightarrow 0 .
$$

By applying the functor $\operatorname{Hom}\left(S_{i}\right.$, ?), we get

$$
\left\langle S_{i}, N\right\rangle_{\tau}+\left\langle S_{i}, G M / N\right\rangle_{\tau}-\left\langle S_{i}, G M\right\rangle_{\tau}+\operatorname{dim} \operatorname{Ext}^{2}\left(S_{i}, N\right) \geq 0 .
$$

By the stable 3 -Calabi-Yau property of $\bmod _{\operatorname{End}}(T)$ proved in 34, we have $\operatorname{dim} \operatorname{Ext}^{2}\left(S_{i}, N\right) \leq \operatorname{dim} \operatorname{Ext}^{1}\left(N, S_{i}\right)$. Therefore, we have

$$
\begin{aligned}
\left.-\operatorname{coind}_{T}(M): T_{i}\right]+\left\langle S_{i}, e\right\rangle_{a} & \geq-\left\langle N, S_{i}\right\rangle_{\tau}-\left\langle S_{i}, G M / N\right\rangle_{\tau}-\operatorname{dim} \operatorname{Ext}^{2}\left(S_{i}, N\right) \\
& \geq-\left[N, S_{i}\right]-\left[S_{i}, G M / N\right]+{ }^{1}\left[S_{i}, G M / N\right] \\
& \geq-m_{i} .
\end{aligned}
$$

6.6. Behaviour of the g-vectors under mutation. Let $B=\left(b_{i j}\right)$ be an antisymmetric integer $r \times r$ matrix. Let $\mathcal{C} \supset$ add $T$ be a triangulated 2-CY realization of $B$. Let $T_{1}, \ldots, T_{r}$ be the nonisomorphic indecomposable factors of $T$. Let $1 \leq l \leq r$ be an integer and $T^{\prime}=\mu_{l}(T)$ the mutation of $T$ at $T_{l}$. Thus, the nonisomorphic indecomposable factors of $T^{\prime}$ are $T_{1}, \ldots, T_{l}^{*}, \ldots, T_{r}$. Let $\mathcal{C}_{1}$ be a principal gluing of $\mathcal{C} \supset$ add $T$ and $\mathcal{C}_{2}$ a principal gluing of $\mathcal{C} \supset$ add $T^{\prime}$ (we assume such gluings exist). For each indecomposable object $M \in \mathcal{C}$ reachable from $T$, we denote by $\mathcal{F}_{M}^{T}$ and $\mathcal{F}_{M}^{T^{\prime}}$ the $\mathcal{F}$-polynomials of $M$ with respect to $\mathcal{C}_{1}$ and $\mathcal{C}_{2}$, respectively. Following [21], we define the integers $h_{l}$ and $h_{l}^{\prime}$ by

$$
\begin{gathered}
u^{h_{l}}=\left.\mathcal{F}_{M}^{T}\right|_{\operatorname{Trop}(u)}\left(u^{\left[-b_{k 1}\right]_{+}}, \ldots, u^{-1}, \ldots, u^{\left[-b_{k n}\right]_{+}}\right), \\
u^{h_{l}^{\prime}}=\left.\mathcal{F}_{M}^{T^{\prime}}\right|_{\operatorname{Trop}(u)}\left(u^{\left[b_{k 1}\right]_{+}}, \ldots, u^{-1}, \ldots, u^{\left[b_{k n}\right]_{+}}\right),
\end{gathered}
$$

where $u^{-1}$ is in the $l$-th position.

The following proposition shows that if the gluings $\mathcal{C}_{1}$ and $\mathcal{C}_{2}$ exist (for example if $\mathcal{C}$ is algebraic and Conjecture 6.1 holds), then Conjecture 6.10 of 21] holds for the cluster algebra with principal coefficients associated with $B$. 
Proposition 6.9. In the above notation, we have

$$
h_{l}^{\prime}=-\left[\left[\operatorname{ind}_{T}(M): T_{l}\right]\right]_{+}, \quad h_{l}=\min \left(0,\left[\operatorname{ind}_{T}(M): T_{l}\right]\right) .
$$

Proof. Let $S_{i}, 1 \leq i \leq r$, be the top of the indecomposable right projective End $_{\mathcal{C}}\left(T^{\prime}\right)$-module $\operatorname{Hom}_{\mathcal{C}}\left(T^{\prime}, T_{i}^{\prime}\right)$. First we will show that $g_{l}=\left[\operatorname{ind}_{T}(M): T_{l}\right]>0$ iff $S_{l}$ occurs as a submodule of the module $\operatorname{Hom}_{\mathcal{C}}\left(T^{\prime}, \Sigma M\right)$ and that the multiplicity of $S_{l}$ in the socle of $\operatorname{Hom}_{\mathcal{C}}\left(T^{\prime}, \Sigma M\right)$ equals $\left[\operatorname{ind}_{T}(M): T_{l}\right]$.

Suppose that $g_{l}>0$. Then we have the following triangle:

$$
T_{M}^{1} \rightarrow T_{M}^{0^{\prime}} \oplus\left(T_{l}\right)^{g_{l}} \rightarrow M \rightarrow \Sigma T_{M}^{1}
$$

with $T_{M}^{1}, T_{M}^{0^{\prime}}$ in add $T$ and $\left[T_{M}^{0^{\prime}}: T_{l}\right]=0$, where $\left(T_{l}\right)^{g_{l}}$ is the sum of $g_{l}$ copies of $T_{l}$. Applying the functor $\operatorname{Hom}_{\mathcal{C}}\left(T^{\prime}, ?\right)$ to the shift of the above triangle, we get the exact sequence

$$
0 \rightarrow \operatorname{Hom}_{\mathcal{C}}\left(T^{\prime}, \Sigma\left(T_{l}\right)^{g_{l}}\right) \rightarrow \operatorname{Hom}_{\mathcal{C}}\left(T^{\prime}, \Sigma M\right) \rightarrow \operatorname{Hom}_{\mathcal{C}}\left(T^{\prime}, \Sigma^{2} T_{M}^{1}\right) \rightarrow \cdots .
$$

Note that $\operatorname{Hom}_{\mathcal{C}}\left(T^{\prime}, \Sigma\left(T_{l}\right)^{g}\right) \cong\left(S_{l}\right)^{g_{l}} ;$ i.e. $S_{l}$ occurs with multiplicity $\geq g_{l}$ in the socle of $\operatorname{Hom}_{\mathcal{C}}\left(T^{\prime}, \Sigma M\right)$. If the multiplicity of $S_{l}$ in the socle of $\operatorname{Hom}_{\mathcal{C}}\left(T^{\prime}, \Sigma M\right)$ was $>g_{l}$, then $S_{l}$ would occur in the socle of $\operatorname{Hom}_{\mathcal{C}}\left(T^{\prime}, \Sigma^{2} T_{M}^{1}\right)$. This is not the case since $\operatorname{Hom}_{\mathcal{C}}\left(T^{\prime}, \Sigma^{2} T_{M}^{1}\right)$ is the sum of injective indecomposables not isomorphic to the injective hull $\operatorname{Hom}_{\mathcal{C}}\left(T^{\prime}, \Sigma^{2} T_{l}\right)$ of $S_{l}$. Conversely, if $S_{l}$ occurs in the socle of $\operatorname{Hom}_{\mathcal{C}}\left(T^{\prime}, \Sigma M\right)$, thanks to the split idempotents property of $\mathcal{C}$, we have an irreducible morphism $\alpha: \Sigma T_{l} \rightarrow \Sigma M$ in $\mathcal{C}$. Thus, by the definition of the index, we get $g_{l}>0$. Moreover, the multiplicity of $S_{l}$ equals $g_{l}$ by the same argument as before.

Assume that $g_{l}>0$. For an arbitrary submodule $U$ of $\operatorname{Hom}_{\mathcal{C}}\left(T^{\prime}, \Sigma M\right)$, let $\operatorname{dim} U=\left(e_{1}, \ldots, e_{n}\right)$. We will show that

$$
e_{l} \leq g_{l}+\sum_{i}\left[b_{i l}\right]_{+} e_{i} .
$$

Indeed, consider the projective resolution of the simple module $S_{l}$ :

$$
\ldots \rightarrow \bigoplus P_{i}^{b_{i l}} \rightarrow P_{l} \rightarrow S_{l} \rightarrow 0 .
$$

Applying the functor $\operatorname{Hom}_{\operatorname{End} d_{\mathcal{C}}\left(T^{\prime}\right)}(?, U)$, we get the exact sequence

$$
0 \rightarrow \operatorname{Hom}\left(S_{l}, U\right) \rightarrow \operatorname{Hom}\left(P_{l}, U\right) \rightarrow \operatorname{Hom}\left(\bigoplus P_{i}^{b_{i l}}, U\right) \rightarrow \ldots,
$$

which implies the inequality because the dimension of $\operatorname{Hom}\left(S_{l}, U\right)$ is less than or equal to the multiplicity of $S_{l}$ in the socle of $\operatorname{Hom}_{\mathcal{C}}\left(T^{\prime}, \Sigma M\right)$, which equals $g_{l}$. By Theorem 6.5, we have

$$
\begin{aligned}
u^{h_{l}^{\prime}} & =\left.\mathcal{F}_{M}^{T^{\prime}}\right|_{\text {Trop }(u)}\left(u^{\left[b_{k 1}\right]_{+}}, \ldots, u^{-1}, \ldots, u^{\left[b_{k n}\right]_{+}}\right) \\
& =1 \oplus \bigoplus_{e} \chi\left(\operatorname{Gr}_{e}\left(\operatorname{Hom}_{\mathcal{C}}\left(T^{\prime}, \Sigma M\right)\right)\right) u^{-e_{l}} \prod_{i \neq l}\left(u^{\left[b_{k i}\right]_{+}}\right)^{e_{i}} .
\end{aligned}
$$

We have just shown that for each $e$, we have

$$
-e_{l}+\sum_{i}\left[b_{i l}\right]_{+} e_{i} \geq g_{l}
$$

and the equality occurs if $e$ is the dimension vector of the submodule $\left(S_{l}\right)^{g_{l}}$. We conclude that we have $h_{l}^{\prime}=-\left[\operatorname{ind}_{T}(M): T_{l}\right]$. If $g_{l} \leq 0$, then $S_{l}$ does not occur in the socle of $\operatorname{Hom}_{\mathcal{C}}\left(T^{\prime}, \Sigma M\right)$ and it is easy to see that $h_{l}^{\prime}=0$. Dually, we have the equality $h_{l}=\min \left(0,\left[\operatorname{ind}_{T}(M): T_{l}\right]\right)$. 
6.7. Acyclic cluster algebras with principal coefficients. Let $B$ be an antisymmetric integer $r \times r$ matrix. Assume that $B$ is acyclic. Let $Q$ be the corresponding quiver of $B$ with the set of vertices $Q_{0}=\{1, \ldots, r\}$ and with the set of arrows $Q_{1}$. Let $\mathcal{C}_{Q}$ be the cluster category of $Q, T=k Q$ the canonical cluster-tilting object of $\mathcal{C}_{Q}$. We claim that the cluster category $\mathcal{C}_{Q} \supset$ add $T$ admits a principal gluing.

Indeed, we define a new quiver $\tilde{Q}=Q \overleftarrow{\coprod} Q_{0}$ associated with $Q$ : its set of vertices is $\{1, \ldots, 2 r\}$, and its arrows are those of $Q$ and new arrows from $r+i$ to $i$ for each vertex $i$ of $Q$. Since $Q$ is acyclic, so is $\tilde{Q}$; hence $k \tilde{Q}$ is finite-dimensional and hereditary. Thus, we have the cluster category $\mathcal{C}_{\tilde{Q}}$ which is a triangulated 2-CY realization of the matrix

$$
\left(\begin{array}{rr}
B & -I_{r} \\
I_{r} & 0
\end{array}\right)
$$

In particular, $\mathcal{C}_{\tilde{Q}} \supset$ add $k \tilde{Q}$ is a principal gluing for $\mathcal{C}_{Q} \supset$ add $T$. Thus, Proposition 6.2. Theorem 6.3. Theorem 6.5 and Proposition 6.6 hold for acyclic cluster algebras with principal coefficients.

Let $P_{i}, 1 \leq i \leq 2 r$, be the nonisomorphic indecomposable projective right modules of $k \tilde{Q}$. Let $\mathcal{P}=\operatorname{add}\left(P_{r+1} \oplus \ldots \oplus P_{2 r}\right)$. We have a triangle equivalence

$$
{ }^{\perp}(\Sigma \mathcal{P}) / \mathcal{P} \stackrel{\sim}{\longrightarrow} \mathcal{C}_{Q}
$$

Recall that there is a partial order on $\mathbb{Z}^{r}$ defined by

$$
\alpha \leq \beta \text { iff } \alpha(i) \leq \beta(i), \text { for } 1 \leq i \leq r, \text { where } \alpha, \beta \in \mathbb{Z}^{r} .
$$

Proposition 6.10. Let $B$ be a $2 r \times r$ integer matrix, whose principal part is antisymmetric and acyclic and whose complementary part is the identity matrix. Let $\sigma$ be a sequence $k_{1}, \ldots, k_{m}$ with $1 \leq k_{i} \leq r$. Denote by $B_{\sigma}$ the matrix

$$
\mu_{k_{1}} \circ \mu_{k_{2}} \ldots \circ \mu_{k_{m}}(B)=\left(b_{i j}^{\sigma}\right) .
$$

Let $E_{\sigma}=\left(e_{1}, e_{2}, \ldots, e_{r}\right)$ be the complementary part of $B_{\sigma}$, where $e_{i} \in \mathbb{Z}^{r}, 1 \leq i \leq r$. Then for each $i$, we have $e_{i} \leq 0$ or $e_{i} \geq 0$.

Proof. Suppose that there is some $k$ such that $e_{k} \not \leq 0$ and $e_{k} \nsucceq 0$. For simplicity, assume that $k=1$, i.e., that there are $r<i, j \leq 2 r$ such that $b_{i 1}^{\sigma}>0$ and $b_{j 1}^{\sigma}<0$.

Let $Q$ be the quiver corresponding to the principal part of $B$ and let $\tilde{Q}$ be as constructed above. By the argument above, there is a cluster-tilting object $T^{\prime}$ of $\mathcal{C}_{k \tilde{Q}}$ such that $B\left(T^{\prime}\right)^{0}=B_{\sigma}$. We have arrows $P_{i} \rightarrow T_{1}^{\prime}$ and $T_{1}^{\prime} \rightarrow P_{j}$, where $T_{1}^{\prime}$ is the indecomposable direct summand of $T^{\prime}$ corresponding to the first column of $B_{\sigma}$. Now if we consider the mutation in direction 1 of $T^{\prime}$, we will have an arrow $P_{i} \rightarrow P_{j}$ in $Q_{\mu_{1}\left(T^{\prime}\right)}$. But this is impossible, since for $r<l \leq 2 r$, the $P_{l}$ are simple pairwise nonisomorphic modules, so we have

$$
\operatorname{Hom}_{\mathcal{C}_{k \tilde{Q}}}\left(P_{i}, P_{j}\right)=\operatorname{Hom}_{k \tilde{Q}}\left(P_{i}, P_{j}\right)=0 .
$$

\section{ACKNowledgments}

We thank A. Zelevinsky for stimulating discussions and for informing us about the ongoing work in [16] and [12]. We are grateful to A. Buan and I. Reiten for sharing the results on dimension vectors obtained in [5. We thank J. Schröer and O. Iyama for their interest and for motivating discussions. The first-named author 
gratefully acknowledges a 5-month fellowship of the Liegrits network (MRTN-CT 2003-505078) during which this research was carried out. Both authors thank the referee for his great help in making this article more readable.

\section{REFERENCES}

[1] Claire Amiot, Cluster categories for algebras of global dimension 2 and quivers with potential, arXiv:0805.1035.

[2] Aslak Bakke Buan, Osamu Iyama, Idun Reiten, and Jeanne Scott, Cluster structures for 2-Calabi-Yau categories and unipotent groups, arXiv:math.RT/0701557, to appear in Compositio Math.

[3] Aslak Bakke Buan, Osamu Iyama, Idun Reiten, and David Smith, Mutation of cluster-tilting objects and potentials, arXiv:0804.3813.

[4] Aslak Bakke Buan and Robert Marsh, Cluster-tilting theory, Trends in representation theory of algebras and related topics, Contemp. Math., vol. 406, Amer. Math. Soc., Providence, RI, 2006, pp. 1-30. MR2258039 (2008f:16031)

[5] Aslak Bakke Buan, Robert Marsh, and Idun Reiten, Denominators of cluster variables, arXiv:0710.4335, to appear in the Journal of the LMS

[6] Aslak Bakke Buan, Robert J. Marsh, Markus Reineke, Idun Reiten, and Gordana Todorov, Tilting theory and cluster combinatorics, Advances in Mathematics 204 (2) (2006), 572-618. MR2249625 (2007f:16033)

[7] Aslak Bakke Buan, Robert J. Marsh, Idun Reiten, and Gordana Todorov, Clusters and seeds in acyclic cluster algebras, Proc. Amer. Math. Soc. 135 (2007), no. 10, 3049-3060 (electronic), With an appendix coauthored in addition by P. Caldero and B. Keller. MR2322734 (2008j:16044)

[8] Arkady Berenstein, Sergey Fomin, and Andrei Zelevinsky, Cluster algebras. III. Upper bounds and double Bruhat cells, Duke Math. J. 126 (2005), no. 1, 1-52. MR2110627(2005i:16065)

[9] Philippe Caldero and Frédéric Chapoton, Cluster algebras as Hall algebras of quiver representations, Comment. Math. Helv. 81 (2006), no. 3, 595-616. MR.2250855 (2008b:16015)

[10] Philippe Caldero and Bernhard Keller, From triangulated categories to cluster algebras. II, Ann. Sci. École Norm. Sup. (4) 39 (2006), no. 6, 983-1009. MR2316979 (2008m:16031)

[11] Philippe Caldero and Bernhard Keller, From triangulated categories to cluster algebras, Invent. Math. 172 (2008), 169-211. MR2385670

[12] Giovanni Cerulli Irelli, Structural theory of rank three cluster algebras of affine type, Ph.D. thesis, Pádova, 2008.

[13] Raika Dehy and Bernhard Keller, On the combinatorics of rigid objects in 2-Calabi-Yau categories, International Mathematics Research Notices 2008 (2008), rnn029-17.

[14] Laurent Demonet, Catégorification d'algèbres amassées antisymétrisables, Ph.D. thesis, Caen, November 2008.

[15] Harm Derksen, Jerzy Weyman, and Andrei Zelevinsky, Quivers with potentials and their representations I: Mutations, arXiv:0704.0649v2, to appear in Selecta Mathematica.

[16] _ Quivers with potentials and their representations II, in preparation.

[17] Grégoire Dupont, Caldero-Keller approach to the denominators of cluster variables, arXiv:0711.4661v1 [math.RT].

[18] V. V. Fock and A. B. Goncharov, Cluster ensembles, quantization and the dilogarithm, arXiv:math.AG/0311245.

[19] Sergey Fomin and Andrei Zelevinsky, Cluster algebras. I. Foundations, J. Amer. Math. Soc. 15 (2002), no. 2, 497-529 (electronic). MR.1887642 (2003f:16050)

[20] _ Cluster algebras. II. Finite type classification, Invent. Math. 154 (2003), no. 1, 63-121. MR.2004457 (2004m:17011)

[21] Cluster algebras IV: Coefficients, Compositio Mathematica 143 (2007), 112-164. MR2295199 (2008d:16049)

[22] P. Gabriel and A.V. Roiter, Representations of finite-dimensional algebras, Encyclopaedia Math. Sci., vol. 73, Springer-Verlag, 1992. MR1239447 (94h:16001b)

[23] Christof Geiß, Bernard Leclerc, and Jan Schröer, Cluster algebra structures and semicanonical bases for unipotent groups, arXiv:math/0703039.

[24] _ Preprojective algebras and cluster algebras, Survey article to appear in the Proceedings of the ICRA XII, arXiv:0804.3168. 
[25] _ Semicanonical bases and preprojective algebras, Ann. Sci. École Norm. Sup. (4) 38 (2005), no. 2, 193-253. MR 2144987 (2007h:17018)

[26] _ Rigid modules over preprojective algebras, Invent. Math. 165 (2006), no. 3, 589-632. MR2242628 (2007g:16023)

[27] Michael Gekhtman, Michael Shapiro, and Alek Vainshtein, Cluster algebras and Poisson geometry, Mosc. Math. J. 3 (2003), no. 3, 899-934, 1199, \{Dedicated to Vladimir Igorevich Arnold on the occasion of his 65th birthday\}. MR2078567(2005i:53104)

[28] _ Cluster algebras and Weil-Petersson forms, Duke Math. J. 127 (2005), no. 2, 291311. MR2130414 (2006d:53103)

[29] Dieter Happel, On the derived category of a finite-dimensional algebra, Comment. Math. Helv. 62 (1987), no. 3, 339-389. MR910167 (89c:16029)

[30] Bernhard Keller, Categorification of acyclic cluster algebras: An introduction, to appear in the proceedings of the conference 'Higher structures in Geometry and Physics 2007', Birkhäuser.

[31] —, Cluster algebras, quiver representations and triangulated categories, arXive:0807.1960.

[32] - Triangulated Calabi-Yau categories, to appear in the proceedings of the workshop of the ICRA 12, Torun, August 2007, available at the author's homepage.

[33] Bernhard Keller and Idun Reiten, Acyclic Calabi-Yau categories are cluster categories, preprint, 2006, with an appendix by Michel Van den Bergh, Compos. Math. 144 (2008), 1332-1348. MR 2457529

[34] Cluster-tilted algebras are Gorenstein and stably Calabi-Yau, Advances in Mathematics 211 (2007), 123-151. MR.2313531 (2008b:18018)

[35] Robert Marsh, Markus Reineke, and Andrei Zelevinsky, Generalized associahedra via quiver representations, Trans. Amer. Math. Soc. 355 (2003), no. 10, 4171-4186 (electronic). MR.1990581 (2004g:52014)

[36] Yann Palu, On algebraic Calabi-Yau categories, Ph.D. thesis, in preparation.

[37] _ Cluster characters for 2-Calabi-Yau triangulated categories, Annales de l'Institut Fourier 58 (2008), no. 6, 2221-2248.

[38] Daniel Quillen, Higher algebraic K-theory. I, Algebraic $K$-theory, I: Higher $K$-theories (Proc. Conf., Battelle Memorial Inst., Seattle, Wash., 1972), Lecture Notes in Math., vol. 341, Springer-Verlag, 1973, pp. 85-147. MR0338129 (49:2895)

[39] Idun Reiten, Tilting theory and cluster algebras, preprint available at www.institut.math. jussieu.fr/ $\sim$ keller/ictp2006/lecturenotes/reiten.pdf.

[40] Claus Michael Ringel, Some remarks concerning tilting modules and tilted algebras. Origin. Relevance. Future., Handbook of Tilting Theory, LMS Lecture Note Series, vol. 332, Cambridge Univ. Press, Cambridge, 2007, pp. 49-104. MR2384619 (2009b:16033)

[41] Joshua S. Scott, Grassmannians and cluster algebras, Proc. London Math. Soc. (3) 92 (2006), no. 2, 345-380. MR.2205721 (2007e:14078)

[42] Michel Van den Bergh, The signs of Serre duality, Appendix A to R. Bocklandt, Graded Calabi-Yau algebras of dimension 3, Journal of Pure and Applied Algebra 212 (2008), 14-32.

Department of Mathematics, Sichuan University, 610064 Chengdu, People's Republic OF CHINA

E-mail address: flyinudream@yahoo.com.cn

U.F.R. De Mathématiques, Institut de Mathématiques, U.M.R. 7586 DU CNRS, UniverSité Paris Diderot - Paris 7, Case 7012, BÂtiment Chevaleret, 75205 Paris Cedex 13, FRANCE

E-mail address: keller@math.jussieu.fr

$U R L$ : www.math.jussieu.fr/ keller 